\title{
INFINITELY MANY SOLUTIONS FOR QUASILINEAR ELLIPTIC EQUATIONS INVOLVING DOUBLE CRITICAL TERMS AND BOUNDARY GEOMETRY
}

\section{Chunhua Wang and Chang-Lin Xiang}

School of Mathematics and Statistics and Hubei Key Laboratory of Mathematical Sciences Central China Normal University, Wuhan 430079, P. R. China; chunhuawang@mail.ccnu.edu.cn

Yangtze University, School of Information and Mathematics, Jingzhou 434023, P. R. China and University of Jyväskylä, Department of Mathematics and Statistics

P. O. Box 35, FI-40014 University of Jyväskylä, Finland; Xiang_math@126.com

Abstract. In this paper, we study the following problem

$$
\begin{cases}-\Delta_{p} u=\mu|u|^{\frac{N p}{N-p}-2} u+\frac{|u|^{\frac{(N-s) p}{N-p}-2} u}{|x|^{s}}+a(x)|u|^{p-2} u & \text { in } \Omega \\ u=0 & \text { on } \partial \Omega\end{cases}
$$

where $1<p<N, 0<s<p, \mu \geq 0$ are constants, $\Delta_{p}$ is the $p$-Laplacian operator, $\Omega \subset \mathbf{R}^{N}$ is a $C^{2}$ bounded domain with $0 \in \bar{\Omega}$ and $a \in C^{1}(\bar{\Omega})$. By an approximation argument, we prove that if $N>p^{2}+p, a(0)>0$ and $\Omega$ satisfies some geometry conditions if $0 \in \partial \Omega$, for example, all the principle curvatures of $\partial \Omega$ at 0 are negative, then the above problem has infinitely many solutions.

\section{Introduction and main results}

In this paper, we study the following quasilinear elliptic problem

$$
\begin{cases}-\Delta_{p} u=\mu|u|^{p^{*}-2} u+\frac{|u|^{p^{*}(s)-2} u}{|x|^{s}}+a(x)|u|^{p-2} u & \text { in } \Omega \\ u=0 & \text { on } \partial \Omega\end{cases}
$$

where $1<p<N, 0<s<p, p^{*}=N p /(N-p), p^{*}(s)=(N-s) p /(N-p)$ and $\mu \geq 0$ are constants,

$$
\Delta_{p} u=\sum_{i=1}^{N} \partial_{x_{i}}\left(|\nabla u|^{p-2} \partial_{x_{i}} u\right), \quad \nabla u=\left(\partial_{x_{1}} u, \cdots, \partial_{x_{N}} u\right)
$$

is the $p$-Laplacian operator, $\Omega \subset \mathbf{R}^{N}$ is a bounded $C^{2}$ domain with $0 \in \bar{\Omega}$ and $a \in C^{1}(\bar{\Omega})$. Equation (1.1) is known as the Euler-Lagrange equation of the energy functional $I: W_{0}^{1, p}(\Omega) \rightarrow \mathbf{R}$ defined as

$$
I(u)=\frac{1}{p} \int_{\Omega}\left(|\nabla u|^{p}-a(x)|u|^{p}\right) d x-\frac{\mu}{p^{*}} \int_{\Omega}|u|^{p^{*}} d x-\frac{1}{p^{*}(s)} \int_{\Omega} \frac{|u|^{p^{*}(s)}}{|x|^{s}} d x
$$

doi:10.5186/aasfm.2016.4161

2010 Mathematics Subject Classification: Primary 35J60, 35B33.

Key words: Quasilinear elliptic equations, double critical terms, boundary geometry condition, infinitely many solutions, approximation argument.

The first-named author is partially supported by NSFC (No. 11301204; No. 11371159; No. 11571130) and self-determined research funds of Central China Normal University from the colleges basic research and operation of MOE(CCNU14A05036). The second-named author is financially supported by the Academy of Finland, project 259224. 
for $u \in W_{0}^{1, p}(\Omega)$. All of the integrals in energy functional $I$ are well defined, due to the Sobolev inequality

$$
\left(\int_{\Omega}|\varphi|^{p^{*}} d x\right)^{p / p^{*}} \leq C \int_{\Omega}|\nabla \varphi|^{p} d x, \quad \forall \varphi \in W_{0}^{1, p}(\Omega),
$$

where $C=C(N, p)>0$, and due to the Caffarelli-Kohn-Nirenberg inequality (see [5])

$$
\left(\int_{\Omega} \frac{|\varphi|^{p^{*}(s)}}{|x|^{s}} d x\right)^{p / p^{*}(s)} \leq C^{\prime} \int_{\Omega}|\nabla \varphi|^{p} d x, \quad \forall \varphi \in W_{0}^{1, p}(\Omega),
$$

where $C^{\prime}=C^{\prime}(N, p, s)>0$.

Equations of type (1.1) has been studied extensively in the literature. A prototype of equation (1.1) is the following semilinear equation

$$
\begin{cases}-\Delta u=|u|^{2^{*}-2} u+\lambda u & \text { in } \Omega \\ u=0 & \text { on } \partial \Omega\end{cases}
$$

where $\lambda>0$ is a constant. As one of their main results, Brézis and Nirenberg [4] proved that equation (1.3) has a positive solution if and only if $0<\lambda<\lambda_{1}$ when $N \geq 4$, or $\lambda^{*}<\lambda<\lambda_{1}$ when $N=3$, where $\lambda_{1}$ is the first eigenvalue of the Laplacian in the domain $\Omega$ with respect to zero Dirichlet boundary condition and $\lambda^{*} \in\left(0, \lambda_{1}\right)$ is a constant. For more existence results, we refer to e.g. $[2,3,8,9,13,15,30]$ on semilinear problems and $[6,11,12,14,16,20,21,26,34]$ on quasilinear problems.

A natural question arises from the results of Brézis and Nirenberg [4] is whether equation (1.3) has infinitely many solutions. This problem has been answered affirmatively by Devillanova and Solimini [15] for all $\lambda>0$, under the assumption that $N>6$, see also the references therein for more related results.

Since we are interested in quasilinear elliptic equations, let us first consider equation (1.1) without the Hardy term $|x|^{-s}|u|^{p^{*}(s)-2} u$. Then equation (1.1) is reduced to

$$
\begin{cases}-\Delta_{p} u=\mu|u|^{p^{*}-2} u+a(x)|u|^{p-2} u & \text { in } \Omega, \\ u=0 & \text { on } \partial \Omega\end{cases}
$$

where $\mu>0$ is assumed. By the same idea of Devillanova and Solimini [15], Cao, Peng and Yan [6] proved the existence of infinitely many solutions to equation (1.4) under the assumption that $N>p^{2}+p$ and $a \equiv$ constant $>0$. We remark that their results can be extended directly to equation (1.4) in the case when $N>p^{2}+p, \mu>0$ and $a \in C^{1}(\bar{\Omega})$ with $a(0)>0$.

Recently, some attention is paid to elliptic problems with double critical terms together with boundary geometry conditions on the domain. For instance, among other problems, Hsia, Lin and Wadade [23] considered the following equation

$$
\begin{cases}-\Delta u=\mu|u|^{2^{*}-2} u+\frac{|u|^{2^{*}(s)-2} u}{|x|^{s}} & \text { in } \Omega \\ u=0 & \text { on } \partial \Omega\end{cases}
$$

where $\mu>0$. Note that equation (1.5) is the special case of equation (1.1) when $p=2$ and $a \equiv 0$. Assuming that $0 \in \partial \Omega$ and the mean curvature of $\partial \Omega$ at 0 is negative, Hsia, Lin and Wadade [23] proved the existence of positive solutions to equation (1.5) for all $0<s<2$ when $N \geq 4$, and for $0<s<1$ when $N=3$. For more results in this respect, we refer to e.g. [10, 18, 19, 24]. 
As to the existence of infinitely many solutions to equations of type (1.1) with double critical terms, to our best knowledge, the first result was obtained by Yan and Yang [33] in the semilinear case $(p=2)$. Note that $|x|^{-s}$ is unbounded in $\Omega$ since we assume that $0 \in \bar{\Omega}$. This brings extra difficulties and requires careful analysis for Yan and Yang to study their problem. Under the assumptions that $N>6, a(0)>0$ and that $\Omega \in C^{3}$ satisfies the following geometry condition:

all the principle curvatures of $\partial \Omega$ at 0 are negative when $0 \in \partial \Omega$,

they proved the existence of infinitely many solutions for equation (1.1) with $p=2$, see [33, Theorem 1.2]. In this paper, our aim is to extend the results of Yan and Yang [33] to the quasilinear setting. That is, we consider equation (1.1) for all $1<p<N$. We will use the same idea as in Yan and Yang [33], which was originally from Devillanova and Solimini [15]. But in the quasilinear setting, it is expected that there are much more complexity that will be encountered than that of Yan and Yang [33]. In the following, we first illustrate the idea that will be used in this paper, and then give the main results of this paper. See also $[6,7,33]$ for more applications of the same idea.

Note that the functional $I$ defined by (1.2) does not satisfy the Palais-Smale condition at large energy level. So it is impossible to apply the mountain pass lemma [1] directly to obtain the existence of infinitely many solutions for equation (1.1). Thus, to derive approximation solutions to equation (1.1), we turn to the following perturbed problem:

$$
\begin{cases}-\Delta_{p} u=\mu|u|^{p^{*}-2-\epsilon} u+\frac{|u|^{*^{*}(s)-2-\epsilon} u}{|x|^{s}}+a(x)|u|^{p-2} u & \text { in } \Omega \\ u=0 & \text { on } \partial \Omega\end{cases}
$$

where $p^{*}(s)-1>\epsilon>0$ is a constant which will tend to zero in the end. The functional corresponding to equation (1.7) is given by

$$
\begin{aligned}
I_{\epsilon}(u)= & \frac{1}{p} \int_{\Omega}\left(|\nabla u|^{p}-a(x)|u|^{p}\right) d x-\frac{\mu}{p^{*}-\epsilon} \int_{\Omega}|u|^{p^{*}-\epsilon} d x \\
& -\frac{1}{p^{*}(s)-\epsilon} \int_{\Omega} \frac{|u|^{p^{*}(s)-\epsilon}}{|x|^{s}} d x
\end{aligned}
$$

for $u \in W_{0}^{1, p}(\Omega)$. Now $I_{\epsilon}$ is an even functional and satisfies the Palais-Smale condition in all energy levels. It follows from the symmetric mountain pass lemma $[1,28]$ that equation (1.7) has infinitely many solutions. See also [17, 20, 27, 29]. Precisely, for fixed $\epsilon, \epsilon>0$, there are positive numbers $c_{l, \epsilon}$ and critical points $u_{l, \epsilon}, l=1,2, \cdots$, such that

$$
I_{\epsilon}\left(u_{l, \epsilon}\right)=c_{l, \epsilon} \rightarrow \infty \quad \text { as } l \rightarrow \infty .
$$

Moreover, for each fixed $l \geq 1$, the sequence $\left\{c_{l, \epsilon}\right\}_{\epsilon>0}$ is bounded and thus can be assumed to converge to a finite limit $c_{l}$ as $\epsilon \rightarrow 0$. To obtain the existence of infinitely many solutions for equation (1.1), the first step is to investigate whether $u_{l, \epsilon}$ converges strongly in $W_{0}^{1, p}(\Omega)$ as $\epsilon \rightarrow 0$ for fixed $l$. That is, we need to study the compactness of the set of solutions for equation (1.7) for all $\epsilon>0$ small. If $u_{l, \epsilon}$ is proven to converge to some $u_{l}$ strongly in $W_{0}^{1, p}(\Omega)$, then $u_{l}$ is a solution to equation $(1.1)$ and $I\left(u_{l}\right)=c_{l}$. The next step is to investigate whether we obtained infinitely many different critical values of $\left\{c_{l}\right\}_{l}$. This step will be disposed via index theory in case $\left\{c_{l}\right\}_{l}$ is a finite set, see e.g. $[6,7,15,17,27,33]$. Both steps being confirmed implies that equation (1.1) admits infinitely many solutions. 
Now it is time to present our assumptions in this paper. Throughout the paper, we use $\|\cdot\|$ to denote the norm of $W_{0}^{1, p}(\Omega)$. We assume that $\Omega$ is a bounded $C^{2}$ domain satisfying the following geometry condition:

$$
x \cdot \nu \leq 0 \text { in a neighborhood of } 0 \text { in } \partial \Omega \text { when } 0 \in \partial \Omega,
$$

where $\nu$ is the outward unit normal of $\partial \Omega$. Examples of domains that satisfy (1.9) will be given in the below. Our main results are the following theorems.

Theorem 1.1. Suppose that $a(0)>0$ and $\Omega \in C^{2}$ satisfies the condition (1.9). If $N>p^{2}+p$, then for any $u_{n}(n=1,2, \cdots)$, which is a solution to equation (1.7) with $\epsilon=\epsilon_{n} \rightarrow 0$, satisfying $\left\|u_{n}\right\| \leq C$ for some constant $C$ independent of $n, u_{n}$ converges strongly in $W_{0}^{1, p}(\Omega)$ up to a subsequence as $n \rightarrow \infty$.

Combining Theorem 1.1 together with index theory, we obtain the following existence result for equation (1.1).

Theorem 1.2. Suppose that $a(0)>0$ and $\Omega \in C^{2}$ satisfies the condition (1.9). If $N>p^{2}+p$, then equation (1.1) has infinitely many solutions.

Note that our assumption (1.9) on the boundary geometry of $\Omega$ when $0 \in \partial \Omega$ is slightly different from (1.6) of Yan and Yang [33]. In fact, our assumption (1.9) is slightly weaker than (1.6). Indeed, suppose that $\Omega \in C^{3}$ and $0 \in \partial \Omega$ such that (1.6) is satisfied. Then we can choose a coordinate system such that

$$
\Omega \cap B_{\delta}(0)=\left\{x: x_{N}>\varphi\left(x^{\prime}\right)\right\} \quad \text { and } \quad \partial \Omega \cap B_{\delta}(0)=\left\{x: x_{N}=\varphi\left(x^{\prime}\right)\right\},
$$

where $\delta>0$ is a small constant and $\varphi:\left\{x^{\prime} \in \mathbf{R}^{N-1}:\left|x^{\prime}\right|<\delta\right\} \rightarrow \mathbf{R}$ is a $C^{3}$ function that has the following expansion at $x^{\prime}=0$ :

$$
\varphi\left(x^{\prime}\right)=-\sum_{j=1}^{N-1} \alpha_{j} x_{j}^{2}+O\left(\left|x^{\prime}\right|^{3}\right) \quad \text { for }\left|x^{\prime}\right| \text { small enough }
$$

with constants $\alpha_{j}>0, j=1, \cdots, N-1$. Then the outward unit normal $\nu$ of $\partial \Omega$ in $B_{\delta}(0)$ is given by

$$
\nu(x)=\frac{\left(\partial_{x_{1}} \varphi\left(x^{\prime}\right), \cdots, \partial_{x_{N-1}} \varphi\left(x^{\prime}\right),-1\right)}{\sqrt{1+\sum_{j=1}^{N-1}\left|\partial_{x_{j}} \varphi\left(x^{\prime}\right)\right|^{2}}} \text { for } x \in \partial \Omega \cap B_{\delta}(0) .
$$

Thus

$$
x \cdot \nu(x)=\frac{-\sum_{j=1}^{N-1} \alpha_{j} x_{j}^{2}+O\left(\left|x^{\prime}\right|^{3}\right)}{\sqrt{1+\sum_{j=1}^{N-1}\left|\partial_{x_{j}} \varphi\left(x^{\prime}\right)\right|^{2}}} \quad \text { for } x \in \partial \Omega \cap B_{\delta}(0),
$$

which implies that

$$
x \cdot \nu(x) \leq 0 \quad \text { for } x \in \partial \Omega \cap B_{\delta^{\prime}}(0),
$$

for some $0<\delta^{\prime}<\delta$ small enough. That is, (1.9) is satisfied. Thus we conclude that assumption (1.6) is slightly stronger than our assumption (1.9).

On the other hand, assumption (1.9) does allow more possibilities than that of (1.6). For instance, consider the case when $\partial \Omega$ has a piece of concave boundary close to 0 if $0 \in \partial \Omega$. Precisely, let $\varphi \in C^{1}$ be such that (1.10) holds, and

$$
0=\varphi(0) \leq \varphi\left(x^{\prime}\right)+\sum_{j=1}^{N-1} \partial_{x_{j}} \varphi\left(x^{\prime}\right)\left(0-x_{j}\right)
$$


for $x^{\prime}$ small enough. Then we have

$$
x \cdot \nu(x)=-\frac{\varphi\left(x^{\prime}\right)+\sum_{j=1}^{N-1} \partial_{x_{j}} \varphi\left(x^{\prime}\right)\left(0-x_{j}\right)}{\sqrt{1+\sum_{j=1}^{N-1}\left|\partial_{x_{j}} \varphi\left(x^{\prime}\right)\right|^{2}}} \leq 0
$$

for $x^{\prime}$ small enough. That is, (1.9) is satisfied. In particular, if $\Omega$ has a piece of flat boundary in a neighborhood of 0 when $0 \in \partial \Omega$, then (1.9) is satisfied, while in such case all the principle curvatures of $\partial \Omega$ vanish in a neighborhood of 0 , which is against (1.6).

Our paper is organized as follows. In Section 2 we establish some integral estimates. In Section 3 we establish estimates for solutions of equation (1.7) in the region which is close to but also suitably away from the blow up point. We prove Theorems 1.1 and 1.2 in Section 4. In order to give a clear line of our framework, we will list some necessary estimates on solutions of quasilinear equation with Hardy potential in Appendix A, a decay estimate for solutions of equations with critical Sobolev growth in Appendix B, some estimates on solutions of $p$-Laplacian equation by Wolff potential in Appendix C, and a global compactness result for the solution $u_{n}$ of equation (1.7) in Appendix D, respectively.

Our notations are standard. $B_{R}(x)$ is the open ball in $\mathbf{R}^{N}$ centered at $x$ with radius $R$. We write

$$
f_{E} u d x=\frac{1}{|E|} \int_{E} u d x
$$

whenever $E$ is a measurable set with $0<|E|<\infty$, the $n$-dimensional Lebesgue measure of $E$. Let $D$ be an arbitrary domain in $\mathbf{R}^{N}$. We denote by $C_{0}^{\infty}(D)$ the space of smooth functions with compact support in $D$. For any $1 \leq r \leq \infty, L^{r}(D)$ is the Banach space of Lebesgue measurable functions $u$ such that the norm

$$
\|u\|_{r, D}= \begin{cases}\left(\int_{D}|u|^{r}\right)^{\frac{1}{r}} & \text { if } 1 \leq r<\infty \\ {\operatorname{ess} \sup _{D}|u|} \text { if } r=\infty\end{cases}
$$

is finite. The local space $L_{\mathrm{loc}}^{r}(D)$ consists of functions belonging to $L^{r}\left(D^{\prime}\right)$ for all $D^{\prime} \subset \subset D$. We also denote $d \mu_{s}=|x|^{-s} d x$ and $\|v\|_{q, \mu_{s}}=\left(\int|v|^{q} d \mu_{s}\right)^{1 / q}$ when there is no confusion on the domain of the integral. A function $u$ belongs to the Sobolev space $W^{1, r}(D)$ if $u \in L^{r}(D)$ and its first order weak partial derivatives also belong to $L^{r}(D)$. We endow $W^{1, r}(D)$ with the norm

$$
\|u\|_{1, r, D}=\|u\|_{r, D}+\|\nabla u\|_{r, D}
$$

The local space $W_{\text {loc }}^{1, r}(D)$ consists of functions belonging to $W^{1, r}\left(D^{\prime}\right)$ for all open $D^{\prime} \subset \subset D$. We recall that $W_{0}^{1, r}(D)$ is the completion of $C_{0}^{\infty}(D)$ in the norm $\|\cdot\|_{1, r, D}$. For the properties of the Sobolev functions, we refer to the monograph [35].

\section{Integral estimates}

Let $u_{n}, n=1,2, \ldots$, be a solution of equation (1.7) with $\epsilon=\epsilon_{n} \rightarrow 0$, satisfying $\left\|u_{n}\right\| \leq C$ for some constant $C$ independent of $n$. In this section we deduce some integral estimates for $u_{n}$. For any function $u$, we define

$$
\rho_{x, \lambda}(u)=\lambda^{\frac{N-p}{p}} u(\lambda(\cdot-x))
$$


for any $\lambda>0$ and $x \in \mathbf{R}^{N}$. By Proposition D.1, $u_{n}$ can be decomposed as

$$
u_{n}=u_{0}+\sum_{j=1}^{m} \rho_{x_{n, j}, \lambda_{n, j}}\left(U_{j}\right)+\omega_{n} .
$$

Here $x_{n, j}=0$ for $j=k+1, \ldots, m$.

To prove that $u_{n}$ converges strongly in $W_{0}^{1, p}(\Omega)$, we only need to show that the bubbles $\rho_{x_{n, j}, \lambda_{n, j}}\left(U_{j}\right)$ will not appear in the decomposition of $u_{n}$. Among all the bubbles, we can choose one bubble such that this bubble has the slowest concentration rate. That is, the corresponding $\lambda$ is the lowest order infinity among all the $\lambda$ appearing in the bubbles. For simplicity, we denote by $\lambda_{n}$ the slowest concentration rate and by $x_{n}$ the corresponding concentration point throughout the paper.

For any $q>1$, denote

$$
\|u\|_{*, q}=\left(\int_{\Omega}|u|^{q} d x\right)^{\frac{1}{q}}+\left(\int_{\Omega}|u|^{\frac{(N-s) q}{N}} d \mu_{s}\right)^{\frac{N}{(N-s) q}}
$$

and $q^{\prime}=q /(q-1)$. Here we write $d \mu_{s}=|x|^{-s} d x$. For any $p^{*} / p^{\prime}<p_{2}<p^{*}<p_{1}$, $\alpha>0$ and $\lambda \geq 1$, consider the following relation

$$
\left\{\begin{array}{l}
\left\|u_{1}\right\|_{*, p_{1}} \leq \alpha \\
\left\|u_{2}\right\|_{*, p_{2}} \leq \alpha \lambda^{\frac{N}{p^{*}-\frac{N}{p_{2}}}}
\end{array}\right.
$$

and define

$$
\|u\|_{*, p_{1}, p_{2}, \lambda}=\inf \alpha
$$

where the infimum is taken over all $\alpha>0$ for which there exist $u_{1}, u_{2}$ such that $|u| \leq u_{1}+u_{2}$ and (2.2) holds. Our main result in this section is the following estimate.

Proposition 2.1. Let $u_{n}, n=1,2, \ldots$, be a solution of equation (1.7) with $\epsilon=\epsilon_{n} \rightarrow 0$, satisfying $\left\|u_{n}\right\| \leq C$ for some positive constant $C$ independent of $n$. Then for any $p_{1}, p_{2} \in\left(p^{*} / p^{\prime}, \infty\right), p_{2}<p^{*}<p_{1}$, there exists a constant $C=C\left(p_{1}, p_{2}\right)>0$, independent of $n$, such that

$$
\left\|u_{n}\right\|_{*, p_{1}, p_{2}, \lambda_{n}} \leq C
$$

for all $n$. Here $\lambda_{n}$ is the slowest concentration rate of $u_{n}$.

Several lemmas are needed to prove Proposition 2.1. In the rest of this section, let us fix a bounded domain $D$ such that $\Omega \subset \subset D$ and set $r=\frac{1}{3} \operatorname{dist}(\Omega, \partial D)$.

Lemma 2.2. Let $w \in W_{0}^{1, p}(D), w \geq 0$, be the solution of

$$
\begin{cases}-\Delta_{p} w=\left(a_{1}(x)+\frac{a_{2}(x)}{|x|^{s}}\right) v^{p-1} & \text { in } D, \\ w=0 & \text { on } \partial D,\end{cases}
$$

where $a_{1}, a_{2}, v \in L^{\infty}(D)$ are nonnegative functions in $D$. Then for any $p_{1}, p_{2} \in$ $\left(p^{*} / p^{\prime}, \infty\right), p_{2}<p^{*}<p_{1}$, there is a constant $C=C\left(p_{1}, p_{2}\right)>0$, such that for any $\lambda \geq 1$

$$
\|w\|_{*, p_{1}, p_{2}, \lambda} \leq C\left(\left\|a_{1}\right\|_{\frac{N}{p}}+\left\|a_{2}\right\|_{\frac{N-s}{p-s}, \mu_{s}}\right)^{\frac{1}{p-1}}\|v\|_{*, p_{1}, p_{2}, \lambda}
$$

Proof. Let $\alpha, \alpha>\|v\|_{*, p_{1}, p_{2}, \lambda}$, be an arbitrary constant. Then by the definition of $\|v\|_{*, p_{1}, p_{2}, \lambda}$, there exist $v_{1}, v_{2}$ such that $|v| \leq v_{1}+v_{2}$ and (2.2) holds with $u_{i}=v_{i}$, $i=1,2$. 
Let $w_{i} \in W_{0}^{1, p}(D), w_{i} \geq 0, i=1,2$, be the solution of equation (2.4) with $v=2 v_{i}$. Then Corollary A.2 implies that

$$
\left\|w_{i}\right\|_{*, p_{i}} \leq C\left(\left\|a_{1}\right\|_{\frac{N}{p}}+\left\|a_{2}\right\|_{\frac{N-s}{p-s}, \mu_{s}}\right)^{\frac{1}{p-1}}\left\|v_{i}\right\|_{*, p_{i}} .
$$

Let $\tilde{w} \in W_{0}^{1, p}(D), \tilde{w} \geq 0$, be the solution of equation

$$
\begin{cases}-\Delta_{p} w=\left(a_{1}(x)+\frac{a_{2}(x)}{|x|^{s}}\right)\left(\left(2 v_{1}\right)^{p-1}+\left(2 v_{2}\right)^{p-1}\right) & \text { in } D, \\ w=0 & \text { on } \partial D .\end{cases}
$$

Applying Corollary A.2 gives us

$$
\begin{aligned}
\|\tilde{w}\|_{*, p_{2}} & \leq C\left(\left\|a_{1}\right\|_{\frac{N}{p}}+\left\|a_{2}\right\|_{\frac{N-s}{p-s}, \mu_{s}}\right)^{\frac{1}{p-1}}\left\|\left(\left(2 v_{1}\right)^{p-1}+\left(2 v_{2}\right)^{p-1}\right)^{\frac{1}{p-1}}\right\|_{*, p_{2}} \\
& \leq C\left(\left\|a_{1}\right\|_{\frac{N}{p}}+\left\|a_{2}\right\|_{\frac{N-s}{p-s}, \mu_{s}}\right)^{\frac{1}{p-1}}\left(\left\|v_{1}\right\|_{*, p_{2}}+\left\|v_{2}\right\|_{*, p_{2}}\right) \\
& \leq C\left(\left\|a_{1}\right\|_{\frac{N}{p}}+\left\|a_{2}\right\|_{\frac{N-s}{p-s}, \mu_{s}}\right)^{\frac{1}{p-1}} \alpha .
\end{aligned}
$$

Thus for any $x \in \Omega$, we have

$$
\inf _{B_{r}(x)} \tilde{w} \leq\left(f_{B_{r}(x)} \tilde{w}^{p_{2}} d y\right)^{\frac{1}{p_{2}}} \leq C\left(\left\|a_{1}\right\|_{\frac{N}{p}}+\left\|a_{2}\right\|_{\frac{N-s}{p-s}, \mu_{s}}\right)^{\frac{1}{p-1}} \alpha .
$$

Note that $v^{p-1} \leq\left(2 v_{1}\right)^{p-1}+\left(2 v_{2}\right)^{p-1}$. Thus $w \leq \tilde{w}$ by comparison principle. Applying Proposition C.1 gives us

$$
w(x) \leq \tilde{w}(x) \leq C \inf _{B_{r}(x)} \tilde{w}+C w_{1}(x)+C w_{2}(x), \quad \forall x \in \Omega .
$$

Let $\tilde{w}_{1}(x)=C \inf _{B_{r}(x)} \tilde{w}+C w_{1}(x)$ and $\tilde{w}_{2}(x)=C w_{2}(x)$ for $x \in \Omega$. Then $w \leq \tilde{w}_{1}+\tilde{w}_{2}$ in $\Omega$. By (2.6) and (2.8), we have that

$$
\left\|\tilde{w}_{1}\right\|_{*, p_{1}} \leq C\left(\left\|a_{1}\right\|_{\frac{N}{p}}+\left\|a_{2}\right\|_{\frac{N-s}{p-s}, \mu_{s}}\right)^{\frac{1}{p-1}} \alpha,
$$

and that

$$
\left\|\tilde{w}_{2}\right\|_{*, p_{2}} \leq C\left(\left\|a_{1}\right\|_{\frac{N}{p}}+\left\|a_{2}\right\|_{\frac{N-s}{p-s}, \mu_{s}}\right)^{\frac{1}{p-1}} \alpha \lambda^{\frac{N}{p^{*}}-\frac{N}{p_{2}}} .
$$

Hence by definition (2.3), we obtain that

$$
\|w\|_{*, p_{1}, p_{2}, \lambda} \leq C\left(\left\|a_{1}\right\|_{\frac{N}{p}}+\left\|a_{2}\right\|_{\frac{N-s}{p-s}, \mu_{s}}\right)^{\frac{1}{p-1}} \alpha .
$$

Since $\alpha>\|v\|_{*, p_{1}, p_{2}, \lambda}$ is arbitrary, we obtain (2.5). The proof of Lemma 2.2 is completed.

We also have the following result which will be used in the proof of Proposition 2.1.

Lemma 2.3. Let $w \in W_{0}^{1, p}(D), w \geq 0$, be the solution of

$$
\begin{cases}-\Delta_{p} w=2 \mu v^{p^{*}-1}+\frac{2 v^{p^{*}(s)-1}}{|x|^{s}}+\frac{A}{|x|^{s}} & \text { in } D, \\ w=0 & \text { on } \partial D,\end{cases}
$$

where $v \geq 0$ is a bounded function and $A \geq 0$ is a constant. Then for any $p_{1}, p_{2} \in$ $\left(p^{*}-1, \frac{\bar{N}}{p}\left(p^{*}-1\right)\right), p_{2}<p^{*}<p_{1}$, and for any $\lambda \geq 1$, there exists a constant 
$C=C\left(p_{1}, p_{2}\right)>0$, such that

$$
\|w\|_{*, q_{1}, q_{2}, \lambda} \leq C\|v\|_{*, p_{1}, p_{2}, \lambda}^{\frac{p^{*}-1}{p-1}}+C
$$

where $q_{1}, q_{2}$ are given by

$$
q_{1}=\frac{(p-1) N \hat{p}_{1}}{N-p \hat{p}_{1}} \quad \text { with } \hat{p}_{1}=\frac{N p_{1}}{\left(p^{*}(s)-1\right) N+s p_{1}},
$$

and

$$
q_{2}=\frac{(p-1) N \hat{p}_{2}}{N-p \hat{p}_{2}} \quad \text { with } \hat{p}_{2}=\frac{p_{2}}{p^{*}-1} .
$$

Proof. Let $\alpha, \alpha>\|v\|_{*, p_{1}, p_{2}, \lambda}$, be an arbitrary constant. Then by the definition of $\|v\|_{*, p_{1}, p_{2}, \lambda}$, there exist $v_{1}, v_{2}$ such that $|v| \leq v_{1}+v_{2}$ and (2.2) holds with $u_{i}=v_{i}$, $i=1,2$.

Let $w_{1} \in W_{0}^{1, p}(D), w_{1} \geq 0$, be the solution of equation (2.9) with $v=2 v_{1}$. Let

$$
\hat{p}_{1}=\min \left\{\frac{p_{1}}{p^{*}-1}, \frac{N p_{1}}{\left(p^{*}(s)-1\right) N+s p_{1}}\right\}
$$

such that $\left(p^{*}-1\right) \hat{p}_{1} \leq p_{1}$ and $\left(p^{*}(s)-1\right) \frac{(N-s) \hat{p}_{1}}{N-s \hat{p}_{1}} \leq \frac{(N-s) p_{1}}{N}$. By the assumptions on the parameters $N, p, s$ and $p_{1}$, we obtain that

$$
\hat{p}_{1}=\frac{N p_{1}}{\left(p^{*}(s)-1\right) N+s p_{1}} \in\left(1, \frac{N}{p}\right) .
$$

Then applying Proposition A.1 gives us

$$
\begin{aligned}
\left\|w_{1}\right\|_{*, q_{1}} & \leq C\left(\left\|v_{1}^{p^{*}-1}\right\|_{\hat{p}_{1}}+\left\|v_{1}^{p^{*}(s)-1}+A\right\|_{\frac{(N-s) \hat{p}_{1}}{N-s \hat{p}_{1}}, \mu_{s}}\right)^{\frac{1}{p-1}} \\
& \leq C\left(\left\|v_{1}\right\|_{p_{1}}^{p^{*}-1}+\left\|v_{1}\right\|_{\frac{(N-s) p_{1}}{N}, \mu_{s}}^{p^{*}(s)-1}+1\right)^{\frac{1}{p-1}} \leq C \alpha^{\frac{p^{*}-1}{p-1}}+C,
\end{aligned}
$$

where $q_{1}=(p-1) N \hat{p}_{1} /\left(N-p \hat{p}_{1}\right)$.

Similarly, let $w_{2} \in W_{0}^{1, p}(D), w_{2} \geq 0$, be the solution of equation

$$
\begin{cases}-\Delta_{p} w_{2}=2 \mu v^{p^{*}-1}+\frac{2 v^{p^{*}(s)-1}}{|x|^{s}} & \text { in } D, \\ w_{2}=0 & \text { on } \partial D .\end{cases}
$$

Let

$$
\hat{p}_{2}=\min \left\{\frac{p_{2}}{p^{*}-1}, \frac{N p_{2}}{\left(p^{*}(s)-1\right) N+s p_{2}}\right\}
$$

such that $\frac{(N-s) \hat{p}_{2}}{N-s \hat{p}_{2}} \leq \frac{(N-s) p_{2}}{N}$ and $\left(p^{*}-1\right) \hat{p}_{2} \leq p_{2}$. Then by the assumptions on the parameters $N, p, s$ and $p_{2}$, we obtain that

$$
\hat{p}_{2}=\frac{p_{2}}{p^{*}-1} \in(1, N / p) .
$$

Applying Proposition A.1 as above, we obtain that

$$
\left\|w_{2}\right\|_{*, q_{2}} \leq\left(C \alpha^{\frac{p^{*}-1}{p-1}}+C\right) \lambda^{\frac{N}{p^{*}}-\frac{N}{q_{2}}}
$$

where $q_{2}=(p-1) N \hat{p}_{2} /\left(N-p \hat{p}_{2}\right)$. To obtain the above estimate, we used the equality that

$$
\left(\frac{N}{p^{*}}-\frac{N}{p_{2}}\right) \frac{p^{*}-1}{p-1}=\frac{N}{p^{*}}-\frac{N}{q_{2}} .
$$


Let $\tilde{w} \in W_{0}^{1, p}(D), \tilde{w} \geq 0$, be the solution of equation

$$
\begin{cases}-\Delta_{p} \tilde{w}=2 \mu\left(\left(2 v_{1}\right)^{p^{*}-1}+\left(2 v_{2}\right)^{p^{*}-1}\right)+2 \frac{\left(2 v_{1}\right)^{p^{*}(s)-1}+\left(2 v_{2}\right)^{p^{*}(s)-1}}{|x|^{s}}+\frac{A}{|x|^{s}} & \text { in } D, \\ \tilde{w}=0 & \text { on } \partial D .\end{cases}
$$

Estimate as that of (2.7). We obtain that

$$
\|\tilde{w}\|_{*, q_{2}} \leq C \alpha^{\frac{p^{*}-1}{p-1}}+C
$$

which implies that

$$
\inf _{B_{r}(x)} \tilde{w} \leq\left(f_{B_{r}(x)} \tilde{w}^{q_{2}} d y\right)^{\frac{1}{q_{2}}} \leq C \alpha^{\frac{p^{*}-1}{p-1}}+C, \quad \forall x \in \Omega .
$$

Note that $w \leq \tilde{w}$ in $\Omega$. Argue as that of Lemma 2.2. We prove Lemma 2.3. This completes the proof.

Now define $u_{n}=0$ in $D \backslash \Omega$. It is easy to see that

$$
\left.|\mu| u\right|^{p^{*}-2-\epsilon} u+\frac{|u|^{p^{*}(s)-2-\epsilon} u}{|x|^{s}}+\left.a(x)|u|^{p-2} u|\leq 2 \mu| u\right|^{p^{*}-1}+\frac{2|u|^{p^{*}(s)-1}+A}{|x|^{s}}
$$

for sufficiently large constant $A>0$. Let $w_{n} \in W_{0}^{1, p}(D), w_{n} \geq 0$, satisfy

$$
\begin{cases}-\Delta_{p} w_{n}=2 \mu\left|u_{n}\right|^{p^{*}-1}+\frac{2\left|u_{n}\right|^{p^{*}(s)-1}}{|x|^{s}}+\frac{A}{|x|^{s}} & \text { in } D \\ w_{n}=0 & \text { on } \partial D .\end{cases}
$$

Then by comparison principle,

$$
\left|u_{n}\right| \leq w_{n} \quad \text { in } D \text {. }
$$

Moreover, note that $\left\|u_{n}\right\| \leq C$. Multiply both sides of equation (2.11) by $w_{n}$ and then integrate on the domain $D$. We easily obtain that

$$
\left\|w_{n}\right\|_{p^{*}}+\left\|w_{n}\right\|_{p^{*}(s), \mu_{s}} \leq C
$$

where $C>0$ is independent of $n$.

To prove Proposition 2.1, it is enough to prove the estimate of Proposition 2.1 for $w_{n}$. We have the following result.

Lemma 2.4. There exist $p_{1}, p_{2} \in\left(p^{*} / p^{\prime}, \infty\right), p_{2}<p^{*}<p_{1}$, and constant $C=$ $C\left(p_{1}, p_{2}\right)>0$, independent of $n$, such that

$$
\left\|w_{n}\right\|_{*, p_{1}, p_{2}, \lambda_{n}} \leq C .
$$

Proof. By Proposition D.1, $u_{n}$ can be decomposed as

$$
u_{n}=u_{0}+\sum_{j=1}^{k} \rho_{x_{n, j}, \lambda_{n, j}}\left(U_{j}\right)+\sum_{j=k+1}^{m} \rho_{0, \lambda_{n, j}}\left(U_{j}\right)+\omega_{n} .
$$

Write $x_{n, j}=0$ for $j=k+1, \ldots, m$. In the following proof, we denote

$$
u_{n, 0}=u_{0}, \quad u_{n, 1}=\sum_{j=1}^{m} \rho_{x_{n, j}, \lambda_{n, j}}\left(U_{j}\right), \quad \text { and } u_{n, 2}=\omega_{n} .
$$

By (2.12), we have

$$
2 \mu\left|u_{n}\right|^{p^{*}-1}+\frac{2\left|u_{n}\right|^{p^{*}(s)-1}}{|x|^{s}}+\frac{A}{|x|^{s}} \leq C \sum_{i=0}^{2}\left(\left|u_{n, i}\right|^{p^{*}-p}+\frac{\left|u_{n, i}\right|^{p^{*}(s)-p}}{|x|^{s}}\right) w_{n}^{p-1}+\frac{A}{|x|^{s}} .
$$


Let $\tilde{w}_{n} \in W_{0}^{1, p}(D), \tilde{w}_{n} \geq 0$, satisfy

$$
\begin{cases}-\Delta_{p} \tilde{w}_{n}=C \sum_{i=0}^{2}\left(\left|u_{n, i}\right|^{p^{*}-p}+\frac{\left|u_{n, i}\right|^{p^{*}(s)-p}}{|x|^{s}}\right) w_{n}^{p-1}+\frac{A}{|x|^{s}} & \text { in } D, \\ \tilde{w}_{n}=0 & \text { on } \partial D .\end{cases}
$$

Comparison principle implies that

$$
w_{n} \leq \tilde{w}_{n} \text { in } D .
$$

Multiply both sides of equation (2.15) by $\tilde{w}_{n}$. By (2.13) we obtain that

$$
\left\|\tilde{w}_{n}\right\|_{p^{*}}+\left\|\tilde{w}_{n}\right\|_{p^{*}(s), \mu_{s}} \leq C \text {. }
$$

Thus we have

$$
\inf _{B_{r}(x)} \tilde{w}_{n} \leq C, \quad \forall x \in \Omega .
$$

Now let $w_{i} \in W_{0}^{1, p}(D), w_{i} \geq 0, i=0,1,2$, be the solution of equation

$$
\begin{cases}-\Delta_{p} w=C\left(\left|u_{n, i}\right|^{p^{*}-p}+\frac{\left|u_{n, i}\right|^{p^{*}(s)-p}}{|x|^{s}}\right) w_{n}^{p-1}+\frac{A \delta_{i 0}}{|x|^{s}} & \text { in } D, \\ w=0 & \text { on } \partial D,\end{cases}
$$

respectively, where $\delta_{00}=1$ and $\delta_{10}=\delta_{20}=0$. Then by Proposition C.1 and (2.17), we obtain that

$$
\tilde{w}_{n}(x) \leq C+C w_{0}(x)+C w_{1}(x)+C w_{2}(x), \quad \forall x \in \Omega .
$$

In the following we estimate $w_{i}, i=0,1,2$, term by term.

First, we use Proposition A.1 to estimate $w_{0}$. Since $0<s<p$, we can choose $q \geq 1$ such that

and that

$$
\frac{s}{N}+\frac{p-1}{p^{*}}<\frac{1}{q}<\frac{p}{N}+\frac{p-1}{p^{*}}=\frac{p^{*}-1}{p^{*}}
$$

Then

$$
q<N / p
$$

$$
\frac{(p-1) N q}{N-p q}>p^{*} \text { and } \quad \frac{(p-1)(N-s) q}{N-s q}<p^{*}(s) .
$$

Let $p_{1}=\frac{(p-1) N q}{N-p q}$. Applying Proposition A.1 to $w_{0}$ gives us

$$
\begin{aligned}
\left\|w_{0}\right\|_{*, p_{1}} & \leq C\left(\left\|\left|u_{n, 0}\right|^{p^{*}-p} w_{n}^{p-1}\right\|_{q}+\left\|\left|u_{n, 0}\right|^{p^{*}(s)-p} w_{n}^{p-1}+A\right\|_{\frac{(N-s) q}{N-s q}, \mu_{s}}\right)^{\frac{1}{p-1}} \\
& \leq C\left(\left\|w_{n}^{p-1}\right\|_{q}+\left\|w_{n}^{p-1}\right\|_{\frac{(N-s) q}{N-s q}, \mu_{s}}+1\right)^{\frac{1}{p-1}} \\
& \leq C\left(\left\|w_{n}\right\|_{(p-1) q}+\left\|w_{n}\right\|_{\frac{(p-1)(N-s) q}{N-s q}, \mu_{s}}+1\right) \\
& \leq C\left(\left\|w_{n}\right\|_{p^{*}}+\left\|w_{n}\right\|_{p^{*}(s), \mu_{s}}+1\right) \leq C .
\end{aligned}
$$

Here in the second inequality we used the boundedness of $u_{n, 0}=u_{0}$ and in the last inequality we used (2.13). So this gives estimate for $w_{0}$.

Next, we use Corollary A.3 to estimate $w_{1}$. We will choose $p_{2}<p^{*}, p_{2}$ close to $p^{*}$ enough such that

$$
\left\|w_{1}\right\|_{*, p_{2}} \leq C \lambda_{n}^{\frac{N}{p^{*}}-\frac{N}{p_{2}}}
$$


Indeed, applying Corollary A.3 to $w_{1}$ gives us that

$$
\left\|w_{1}\right\|_{*, p_{2}} \leq C\left(\left\|\left|u_{n, 1}\right|^{p^{*}-p}\right\|_{r_{1}}+\left\|\left|u_{n, 1}\right|^{p^{*}(s)-p}\right\|_{r_{2}, \mu_{s}}\right)^{\frac{1}{p-1}}\left\|w_{n}\right\|_{*, p^{*}},
$$

where $r_{1}, r_{2}$ are defined as in (A.4), that is,

$$
\frac{1}{r_{1}}=(p-1)\left(\frac{1}{p_{2}}-\frac{1}{p^{*}}\right)+\frac{p}{N} \text { and } \quad \frac{1}{r_{2}}=(p-1)\left(\frac{N}{(N-s) p_{2}}-\frac{1}{p^{*}(s)}\right)+\frac{p-s}{N-s} .
$$

By (2.13), we have

$$
\left\|w_{1}\right\|_{*, p_{2}} \leq C\left(\left\|\left|u_{n, 1}\right|^{p^{*}-p}\right\|_{r_{1}}+\left\|\left|u_{n, 1}\right|^{p^{*}(s)-p}\right\|_{r_{2}, \mu_{s}}\right)^{\frac{1}{p-1}} .
$$

We only need to estimate $\left\|\left|u_{n, 1}\right|^{p^{*}-p}\right\|_{r_{1}}$ and $\left\|\left|u_{n, 1}\right|^{p^{*}(s)-p}\right\|_{r_{2}, \mu_{s}}$.

For all $1 \leq j \leq m$, we have

$$
\int_{\mathbf{R}^{N}}\left|\rho_{x_{n, j}, \lambda_{n, j}}\left(U_{j}\right)\right|^{\left(p^{*}-p\right) r_{1}} d y=\lambda_{n, j}^{p r_{1}-N} \int_{\mathbf{R}^{N}}\left|U_{j}\right|^{\left(p^{*}-p\right) r_{1}} d y .
$$

By Proposition B.1, for all $1 \leq j \leq m$,

$$
\left|U_{j}(y)\right| \leq \frac{C}{1+|y|^{\frac{N-p}{p-1}}}, \quad \forall y \in \mathbf{R}^{N} .
$$

Since $\frac{N-p}{p-1}\left(p^{*}-p\right) r_{1} \rightarrow \frac{p N}{p-1}$ as $p_{2} \rightarrow p^{*}$, we can choose $p_{2}$ close to $p^{*}$ enough such that $\frac{N-p}{p-1}\left(p^{*}-p\right) r_{1}>N$. Then

$$
\int_{\mathbf{R}^{N}}\left|U_{j}\right|^{\left(p^{*}-p\right) r_{1}} d y<\infty
$$

Thus for all $1 \leq j \leq m$,

$$
\int_{\mathbf{R}^{N}}\left|\rho_{x_{n, j}, \lambda_{n, j}}\left(U_{j}\right)\right|^{\left(p^{*}-p\right) r_{1}} d y \leq C \lambda_{n, j}^{p r_{1}-N}
$$

Therefore

$$
\begin{aligned}
\left\|\left|u_{n, 1}\right|^{p^{*}-p}\right\|_{r_{1}}^{\frac{1}{p-1}} & =\left\|u_{n, 1}\right\|_{\left(p^{*}-p\right) r_{1}}^{\frac{p^{*}-p}{p-1}} \leq C \sum_{j=1}^{m}\left\|\rho_{x_{n, j}, \lambda_{n, j}}\left(U_{j}\right)\right\|_{\left(p^{*}-p\right) r_{1}}^{\frac{p^{*}-p}{p-1}} \\
& \leq C \sum_{j=1}^{m} \lambda_{n, j}^{\frac{p r_{1}-N}{\left(p^{*}-p\right) r_{1}} \cdot \frac{p^{*}-p}{p-1}} \leq C \lambda_{n}^{\frac{N}{p^{*}}-\frac{N}{p_{2}}} .
\end{aligned}
$$

We used the equality

$$
\frac{p r_{1}-N}{\left(p^{*}-p\right) r_{1}} \cdot \frac{p^{*}-p}{p-1}=\frac{N}{p^{*}}-\frac{N}{p_{2}}
$$

in the last inequality of (2.22). This gives estimate for $\left\|\left|u_{n, 1}\right|^{p^{*}-p}\right\|_{r_{1}}$.

We can also choose $p_{2}$ close to $p^{*}$ enough such that for all $1 \leq j \leq m$,

$$
\int_{\mathbf{R}^{N}}\left|\rho_{x_{n, j}, \lambda_{n, j}}\left(U_{j}\right)\right|^{\left(p^{*}(s)-p\right) r_{2}} d \mu_{s} \leq C \lambda_{n, j}^{(p-s) r_{2}-N+s} .
$$

Indeed, we have

$$
\int_{\mathbf{R}^{N}}\left|\rho_{x_{n, j}, \lambda_{n, j}}\left(U_{j}\right)\right|^{\left(p^{*}(s)-p\right) r_{2}} d \mu_{s}=\lambda_{n, j}^{(p-s) r_{2}-N+s} \int_{\mathbf{R}^{N}} \frac{\left|U_{j}(y)\right|^{\left(p^{*}(s)-p\right) r_{2}}}{\left|y+\lambda_{n, j} x_{n, j}\right|^{s}} d y .
$$


Write $y_{n, j}=-\lambda_{n, j} x_{n, j}$. Let

$$
I_{1}=\int_{B_{1}\left(y_{n, j}\right)} \frac{\left|U_{j}(y)\right|^{\left(p^{*}(s)-p\right) r_{2}}}{\left|y-y_{n, j}\right|^{s}} d y \quad \text { and } \quad I_{2}=\int_{\mathbf{R}^{N} \backslash B_{1}\left(y_{n, j}\right)} \frac{\left|U_{j}(y)\right|^{\left(p^{*}(s)-p\right) r_{2}}}{\left|y-y_{n, j}\right|^{s}} d y .
$$

Since $U_{j}$ is bounded and $0<s<N$, we have

$$
I_{1} \leq C \text {. }
$$

Let $\delta>0$ be a number to be determined. By Hölder's inequality, we have

$$
\begin{aligned}
I_{2} & \leq\left(\int_{\mathbf{R}^{N} \backslash B_{1}\left(y_{n, j}\right)} \frac{1}{\left|y-y_{n, j}\right|^{N+\delta}} d y\right)^{\frac{s}{N+\delta}}\left(\int_{\mathbf{R}^{N} \backslash B_{1}\left(y_{n, j}\right)}\left|U_{j}(y)\right|^{\frac{\left(p^{*}(s)-p\right) r_{2}(N+\delta)}{N+\delta-s}} d y\right)^{\frac{N+\delta-s}{N+\delta}} \\
& \leq C_{\delta}\left(\int_{\mathbf{R}^{N}}\left|U_{j}(y)\right|^{\frac{\left(p^{*}(s)-p\right) r_{2}(N+\delta)}{N+\delta-s}} d y\right)^{\frac{N+\delta-s}{N+\delta}} .
\end{aligned}
$$

Since

$$
\frac{N-p}{p-1} \frac{\left(p^{*}(s)-p\right) r_{2}(N+\delta)}{N+\delta-s} \rightarrow \frac{p(N-s)(N+\delta)}{(p-1)(N+\delta-s)} \quad \text { as } p_{2} \rightarrow p^{*},
$$

and

$$
\frac{p(N-s)(N+\delta)}{(p-1)(N+\delta-s)}>N \quad \text { for } \delta>0 \text { small enough, }
$$

we can choose $p_{2}$ close to $p^{*}$ enough and $\delta>0$ small enough such that

$$
\frac{N-p}{p-1} \frac{\left(p^{*}(s)-p\right) r_{2}(N+\delta)}{N+\delta-s}>N \text {. }
$$

Then

and we obtain

$$
\int_{\mathbf{R}^{N}}\left|U_{j}(y)\right|^{\frac{\left(p^{*}(s)-p\right) r_{2}(N+\delta)}{N+\delta-s}} d y<\infty,
$$

$$
I_{2} \leq C
$$

Combining the estimates of $I_{1}$ and $I_{2}$ yields

Hence we have

$$
\int_{\mathbf{R}^{N}}\left|\rho_{x_{n, j}, \lambda_{n, j}}\left(U_{j}\right)\right|^{\left(p^{*}(s)-p\right) r_{2}} d \mu_{s} \leq C \lambda_{n, j}^{(p-s) r_{2}-N+s} .
$$

$$
\begin{aligned}
\left\|\left|u_{n, 1}\right|^{p^{*}(s)-p}\right\|_{r_{2}, \mu_{s}}^{\frac{1}{p-1}} & =\left\|u_{n, 1}\right\|_{\left(p^{*}(s)-p\right) r_{2}, \mu_{s}}^{\frac{p^{*}(s)-p}{p-1}} \leq C \sum_{j=1}^{m} \lambda_{n, j}^{\frac{(p-s) r_{2}-N+s}{\left(p^{*}(s)-p\right) r_{2}} \cdot \frac{p^{*}(s)-p}{p-1}} \\
& \leq C \lambda_{n}^{\frac{N}{p^{*}}-\frac{N}{p_{2}}}
\end{aligned}
$$

In the above inequality we used the equality

$$
\frac{(p-s) r_{2}-N+s}{\left(p^{*}(s)-p\right) r_{2}} \cdot \frac{p^{*}(s)-p}{p-1}=\frac{N}{p^{*}}-\frac{N}{p_{2}} .
$$

Combining (2.21)-(2.23) gives (2.20).

Finally, we use Lemma 2.2 to estimate $w_{2}$. By Lemma 2.2, we have

$$
\begin{aligned}
\left\|w_{2}\right\|_{*, p_{1}, p_{2}, \lambda_{n}} & \leq C\left(\left\|\left|u_{n, 2}\right|^{p^{*}-p}\right\|_{\frac{N}{p}}+\left\|\left|u_{n, 2}\right|^{p^{*}(s)-p}\right\|_{\frac{N-s}{p-s}, \mu_{s}}\right)^{\frac{1}{p-1}}\left\|w_{n}\right\|_{*, p_{1}, p_{2}, \lambda_{n}} \\
& \leq \frac{1}{2 C^{\prime}}\left\|w_{n}\right\|_{*, p_{1}, p_{2}, \lambda_{n}}
\end{aligned}
$$

since $\omega_{n} \rightarrow 0$ in $W_{0}^{1, p}(\Omega)$, where the constant $C^{\prime}$ is given by (2.18). 
Now combining (2.13), (2.18)-(2.20) and (2.24), we obtain that

$$
\begin{aligned}
\left\|\tilde{w}_{n}\right\|_{*, p_{1}, p_{2}, \lambda_{n}} & \leq C+C\left\|w_{0}\right\|_{*, p_{1}, p_{2}, \lambda_{n}}+C\left\|w_{1}\right\|_{*, p_{1}, p_{2}, \lambda_{n}}+C\left\|w_{2}\right\|_{*, p_{1}, p_{2}, \lambda_{n}} \\
& \leq C+C\left\|w_{0}\right\|_{*, p_{1}}+C\left\|w_{1}\right\|_{*, p_{2}}+\frac{1}{2}\left\|w_{n}\right\|_{*, p_{1}, p_{2}, \lambda_{n}} \\
& \leq C+\frac{1}{2}\left\|\tilde{w}_{n}\right\|_{*, p_{1}, p_{2}, \lambda_{n}},
\end{aligned}
$$

which completes the proof.

Now we can prove Proposition 2.1.

Proof of Proposition 2.1. Recall that $w_{n}$ is a solution to equation (2.11). It is standard to prove Proposition 2.1 by Lemma 2.2 and Lemma 2.3. See details in e.g. [6]. This finishes the proof of Proposition 2.1.

\section{Estimates on safe regions}

Since the number of the bubbles of $u_{n}$ is finite, by Proposition D.1 we can always find a constant $\bar{C}>0$, independent of $n$, such that the region

$$
\mathcal{A}_{n}^{1}=\left(B_{(\bar{C}+5) \lambda_{n}^{-\frac{1}{p}}}\left(x_{n}\right) \backslash B_{\bar{C} \lambda_{n}^{-\frac{1}{p}}}\left(x_{n}\right)\right) \cap \Omega
$$

does not contain any concentration point of $u_{n}$ for any $n$. We call this region a safe region for $u_{n}$. Also denote

$$
\mathcal{A}_{n}^{2}=\left(B_{(\bar{C}+4) \lambda_{n}^{-\frac{1}{p}}}\left(x_{n}\right) \backslash B_{(\bar{C}+1) \lambda_{n}^{-\frac{1}{p}}}\left(x_{n}\right)\right) \cap \Omega .
$$

In this section, we prove the following result.

Proposition 3.1. Let $u_{n}, n=1,2, \cdots$, be a solution of equation (1.7) with $\epsilon=\epsilon_{n} \rightarrow 0$, satisfying $\left\|u_{n}\right\| \leq C$ for some positive constant $C$ independent of $n$. Then for any $q \geq p$, there is a constant $C>0$ independent of $n$, such that

$$
\int_{\mathcal{A}_{n}^{2}}\left|u_{n}\right|^{q} d x \leq C \lambda_{n}^{-\frac{N}{p}}
$$

In order to prove Proposition 3.1, we need the following lemma.

Lemma 3.2. Let $D$ be a bounded domain with $\Omega \subset \subset D$ and let $w_{n}$ be the solution of equation (2.11). Then there exist a number $\gamma>p-1$ and a constant $C>0$ independent of $n$, such that

$$
\left(\frac{1}{r^{N}} \int_{B_{r}(y) \cap \Omega} w_{n}^{\gamma} d x\right)^{\frac{1}{\gamma}} \leq C, \quad \forall y \in \Omega,
$$

for all $r \geq \bar{C} \lambda_{n}^{-\frac{1}{p}}$.

Proof. We will combine Proposition 2.1 and Proposition C.2 to prove Lemma 3.2. Since $w_{n}$ is the solution of equation (2.11), applying Proposition C.2 gives us a number $\gamma \in(p-1,(p-1) N /(N-p+1))$ and a constant $C=C(N, p, \gamma)$ such that

$$
\begin{aligned}
\left(\frac{1}{r^{N}} \int_{B_{r}(y) \cap \Omega} w_{n}^{\gamma}\right)^{\frac{1}{\gamma}} & \leq C+C \int_{r}^{R}\left(\int_{B_{t}(y)}\left(2 \mu\left|u_{n}\right|^{p^{*}-1}+\frac{2\left|u_{n}\right|^{p^{*}(s)-1}}{|x|^{s}}+\frac{A}{|x|^{s}}\right)\right)^{\frac{1}{p-1}} \frac{d t}{t^{\frac{N-1}{p-1}}} \\
& \leq C+C \int_{r}^{R}\left(\frac{1}{t^{N-p}} \int_{B_{t}(y)}\left(\left|u_{n}\right|^{p^{*}-1}+\frac{\left|u_{n}\right|^{p^{*}(s)-1}}{|x|^{s}}\right)\right)^{\frac{1}{p-1}} \frac{d t}{t},
\end{aligned}
$$


for all $0<r<R$, where $R=\operatorname{dist}(\Omega, \partial D)$. Let

$$
I_{1}=\int_{r}^{R}\left(\frac{1}{t^{N-p}} \int_{B_{t}(y)}\left|u_{n}\right|^{p^{*}-1} d x\right)^{\frac{1}{p-1}} \frac{d t}{t}
$$

and

$$
I_{2}=\int_{r}^{R}\left(\frac{1}{t^{N-p}} \int_{B_{t}(y)} \frac{\left|u_{n}\right|^{p^{*}(s)-1}}{|x|^{s}} d x\right)^{\frac{1}{p-1}} \frac{d t}{t}
$$

such that

$$
\left(\frac{1}{r^{N}} \int_{B_{r}(y) \cap \Omega} w_{n}^{\gamma} d x\right)^{\frac{1}{\gamma}} \leq C+C I_{1}+C I_{2} .
$$

We now estimate $I_{1}$ and $I_{2}$ for $r \geq \bar{C} \lambda_{n}^{-1 / p}$.

By Proposition 2.1, $\left\|u_{n}\right\|_{*, p_{1}, p_{2}, \lambda} \leq C$ for any $p_{1}, p_{2} \in\left(p^{*} / p^{\prime}, \infty\right), p_{2}<p^{*}<p_{1}$. Let $p_{1}>p^{*}$ be a number to be determined and $p_{2}=p^{*}-1$. There exist $u_{n, 1}, u_{n, 2}$ with $\left|u_{n}\right| \leq u_{n, 1}+u_{n, 2}$ such that $\left\|u_{n, 1}\right\|_{*, p_{1}} \leq C$ and $\left\|u_{n, 2}\right\|_{*, p_{2}} \leq C \lambda_{n}^{\frac{N}{p^{*}}-\frac{N}{p_{2}}}$. Then

$$
\int_{B_{t}(y)}\left|u_{n, 1}\right|^{p^{*}-1} d x \leq C\left(\int_{B_{t}(y)}\left|u_{n, 1}\right|^{p_{1}} d x\right)^{\frac{p^{*}-1}{p_{1}}}\left|B_{t}(y)\right|^{1-\frac{p^{*}-1}{p_{1}}} \leq C t^{\left(1-\frac{p^{*}-1}{p_{1}}\right) N},
$$

and

$$
\int_{B_{t}(y)}\left|u_{n, 2}\right|^{p^{*}-1} d x=\int_{B_{t}(y)}\left|u_{n, 2}\right|^{p_{2}} d x \leq C \lambda_{n}^{\left(\frac{N}{\left.p^{*}-\frac{N}{p_{2}}\right) p_{2}}\right.}=C \lambda_{n}^{\frac{p-N}{p}} .
$$

Thus

$$
\begin{aligned}
\int_{B_{t}(y)}\left|u_{n}\right|^{p^{*}-1} d x & \leq C \int_{B_{t}(y)}\left|u_{n, 1}\right|^{p^{*}-1} d x+C \int_{B_{t}(y)}\left|u_{n, 2}\right|^{p^{*}-1} d x \\
& \leq C t^{\left(1-\frac{p^{*}-1}{p_{1}}\right) N}+C \lambda_{n}^{\frac{p-N}{p}} .
\end{aligned}
$$

Since $\frac{N}{p-1}\left(1-\frac{p^{*}-1}{p_{1}}\right)+\frac{p-N}{p-1} \rightarrow \frac{p}{p-1}$ as $p_{1} \rightarrow \infty$, we can choose $p_{1}$ large enough such that $\frac{N}{p-1}\left(1-\frac{p^{*}-1}{p_{1}}\right)+\frac{p-N}{p-1}>0$. Then

$$
\int_{0}^{R} t^{\left(1-\frac{p^{*}-1}{p_{1}}\right) \frac{N}{p-1}+\frac{p-N}{p-1}} \frac{d t}{t}<C
$$

Note also that for $r \geq \bar{C} \lambda_{n}^{-1 / p}$, we have

$$
\int_{r}^{\infty} t^{\frac{p-N}{p-1}} \frac{d t}{t} \leq C \lambda_{n}^{\frac{N-p}{p(p-1)}}
$$

Therefore

$$
\begin{aligned}
I_{1} & \leq \int_{r}^{R}\left(C t^{\left(1-\frac{p^{*}-1}{p_{1}}\right) N}+C \lambda_{n}^{\frac{p-N}{p}}\right)^{\frac{1}{p-1}} t^{\frac{p-N}{p-1}} \frac{d t}{t} \\
& \leq C \int_{0}^{R} t^{\frac{N}{p-1}\left(1-\frac{p^{*}-1}{p_{1}}\right)+\frac{p-N}{p-1}} \frac{d t}{t}+C \lambda_{n}^{\frac{p-N}{p(p-1)}} \int_{r}^{\infty} t^{\frac{p-N}{p-1}} \frac{d t}{t} \leq C .
\end{aligned}
$$

This gives estimate for $I_{1}$. 
Next we estimate $I_{2}$. Let $\bar{p}_{1}>p^{*}$ be a number to be determined and $\bar{p}_{2}=$ $N\left(p^{*}(s)-1\right) /(N-s)$. There exist $\bar{u}_{n, 1}, \bar{u}_{n, 2}$ with $\left|u_{n}\right| \leq \bar{u}_{n, 1}+\bar{u}_{n, 2}$ such that $\left\|\bar{u}_{n, 1}\right\|_{*, \bar{p}_{1}} \leq C$ and $\left\|\bar{u}_{n, 2}\right\|_{*, \bar{p}_{2}} \leq C \lambda_{n}^{\frac{N}{p^{*}-\frac{N}{\bar{p}_{2}}}}$. Then

$$
\begin{aligned}
\int_{B_{t}(y)}\left|\bar{u}_{n, 1}\right|^{p^{*}(s)-1} d \mu_{s} & \leq\left(\int_{B_{t}(y)}\left|\bar{u}_{n, 1}\right|^{\frac{N-s}{N} \bar{p}_{1}} d \mu_{s}\right)^{\frac{\left(p^{*}(s)-1\right) N}{(N-s) \bar{p}}}\left(\int_{B_{t}(y)} d \mu_{s}\right)^{1-\frac{\left(p^{*}(s)-1\right) N}{(N-s) \bar{p}_{1}}} \\
& \leq C t^{N-s-\frac{\left(p^{*}(s)-1\right) N}{\bar{p}_{1}}}
\end{aligned}
$$

and

$$
\int_{B_{t}(y)}\left|\bar{u}_{n, 2}\right|^{p^{*}(s)-1} d \mu_{s}=\int_{B_{t}(y)}\left|\bar{u}_{n, 2}\right|^{\frac{N-s}{N} \bar{p}_{2}} d \mu_{s} \leq C \lambda_{n}^{\frac{p-N}{p}} .
$$

Arguing as above yields

$$
I_{2} \leq C
$$

if we choose $\bar{p}_{1}$ large enough. This gives estimate for $I_{2}$.

By (3.2)-(3.4), we obtain (3.1). The proof of Lemma 3.2 is complete.

Now we can prove Proposition 3.1.

Proof of Proposition 3.1. Let $\gamma>p-1$ be as in Lemma 3.2. Since $\left|u_{n}\right| \leq w_{n}$, we have

$$
\int_{B_{\lambda_{n}^{-1 / p}}(y)}\left|u_{n}\right|^{\gamma} d x \leq C \lambda_{n}^{-\frac{N}{p}}, \quad \forall y \in \mathcal{A}_{n}^{2} .
$$

Let $v_{n}(x)=u_{n}\left(\lambda_{n}^{-\frac{1}{p}} x\right), x \in \Omega_{n}=\left\{x ; \lambda_{n}^{-\frac{1}{p}} x \in \Omega\right\}$. Then $v_{n}$ is a solution to equation

$$
\begin{cases}-\Delta_{p} v_{n}=\lambda_{n}^{-1}\left(\mu\left|v_{n}\right|^{p^{*}-p-\epsilon_{n}}+\frac{\lambda_{n}^{\frac{s}{p}}\left|v_{n}\right|^{p^{*}(s)-p-\epsilon_{n}}}{|x|^{s}}+a\left(\lambda_{n}^{-\frac{1}{p}} x\right)\right)\left|v_{n}\right|^{p-2} v_{n} & x \in \Omega_{n}, \\ v_{n}=0 & \text { on } \partial \Omega_{n} .\end{cases}
$$

Let $z=\lambda_{n}^{\frac{1}{p}} y, y \in \mathcal{A}_{n}^{2}$. Since $B_{\lambda_{n}^{-1 / p}}(y)$ does not contain any concentration point of $u_{n}$, we can deduce that

$$
\begin{aligned}
\int_{B_{1}(z)}\left|\lambda_{n}^{-1}\left(\mu\left|v_{n}\right|^{p^{*}-p-\epsilon_{n}}+a\left(\lambda_{n}^{-\frac{1}{p}} x\right)\right)\right|^{\frac{N}{p}} d x & \leq C \int_{B_{1}(z)}\left|\lambda_{n}^{-1}\left(\left|v_{n}\right|^{p^{*}-p}+1\right)\right|^{\frac{N}{p}} d x \\
& \leq C \int_{B_{\lambda_{n}^{-\frac{1}{p}}}(y)}\left|u_{n}\right|^{p^{*}} d x+C \lambda_{n}^{-\frac{N}{p}} \rightarrow 0
\end{aligned}
$$

and that

$$
\begin{aligned}
\int_{B_{1}(z)} \frac{\left.\left.\left|\lambda_{n}^{-1} \lambda_{n}^{\frac{s}{p}}\right| v_{n}\right|^{p^{*}(s)-p-\epsilon_{n}}\right|^{\frac{N-s}{p-s}}}{|x|^{s}} d x & \leq C \int_{B_{1}(z)} \frac{\left|\lambda_{n}^{\frac{s-p}{p}}\left(\left|v_{n}\right|^{p^{*}(s)-p}+1\right)\right|^{\frac{N-s}{p-s}}}{|x|^{s}} d x \\
& \leq C \int_{B_{\lambda_{n}-\frac{1}{p}}(y)} \frac{\left|u_{n}\right|^{p^{*}(s)}}{|x|^{s}} d x+C \lambda_{n}^{-\frac{N-s}{p}} \rightarrow 0
\end{aligned}
$$

as $n \rightarrow \infty$. 
Thus for any $q>p^{*}$, we obtain by Lemma A.4 and (3.5) that,

$$
\left\|v_{n}\right\|_{q, B_{1 / 2}(z)} \leq C\left(\int_{B_{1}(z)}\left|v_{n}\right|^{\gamma} d x\right)^{\frac{1}{\gamma}}=C\left(f_{B_{\lambda_{n}^{-1 / p}}(y)}\left|u_{n}\right|^{\gamma} d x\right)^{\frac{1}{\gamma}} \leq C .
$$

Equivalently, we arrive at

$$
\int_{B_{\frac{1}{2} \lambda_{n}^{-1 / p}(y)}}\left|u_{n}\right|^{q} d x \leq C \lambda_{n}^{-\frac{N}{p}}, \quad \forall y \in \mathcal{A}_{n}^{2} .
$$

Now by a covering argument we proves Proposition 3.1 in the case when $q>p^{*}$.

For $p \leq q \leq p^{*}$, we apply Hölder's inequality to obtain that

$$
\left(f_{\mathcal{A}_{n}^{2}}\left|u_{n}\right|^{q} d x\right)^{\frac{1}{q}} \leq\left(f_{\mathcal{A}_{n}^{2}}\left|u_{n}\right|^{2 p^{*}} d x\right)^{\frac{1}{2 p^{*}}} \leq C .
$$

The proof of Proposition 3.1 is complete.

Let

$$
\mathcal{A}_{n}^{3}=\left(B_{(\bar{C}+3) \lambda_{n}^{-\frac{1}{p}}}\left(x_{n}\right) \backslash B_{(\bar{C}+2) \lambda_{n}^{-\frac{1}{p}}}\left(x_{n}\right)\right) \cap \Omega .
$$

In the end of this section, we prove the following gradient estimate for $u_{n}$.

Proposition 3.3. We have

$$
\int_{\mathcal{A}_{n}^{3}}\left|\nabla u_{n}\right|^{p} d x \leq C \int_{\mathcal{A}_{n}^{2}}\left(\left|u_{n}\right|^{p^{*}}+\frac{\left|u_{n}\right|^{p^{*}(s)}}{|x|^{s}}+1\right) d x+C \lambda_{n} \int_{\mathcal{A}_{n}^{2}}\left|u_{n}\right|^{p} d x .
$$

In particular, we have

$$
\int_{\mathcal{A}_{n}^{3}}\left|\nabla u_{n}\right|^{p} d x \leq C \lambda_{n}^{\frac{p-N}{p}}
$$

Proof. Let $\phi \in C_{0}^{\infty}\left(\mathcal{A}_{n}^{2}\right)$ be a cut-off function with $\phi=1$ in $\mathcal{A}_{n}^{3}, 0 \leq \phi \leq 1$ and $|\nabla \phi| \leq C \lambda_{n}^{\frac{1}{p}}$. Multiply the equation of $u_{n}$ by $\phi^{p} u_{n}$ and integrate on the domain $\mathcal{A}_{n}^{2}$. We obtain that

$$
\int_{\mathcal{A}_{n}^{2}}\left|\nabla u_{n}\right|^{p-2} \nabla u_{n} \cdot \nabla\left(\phi^{p} u_{n}\right) d x=\int_{\mathcal{A}_{n}^{2}}\left(\mu\left|u_{n}\right|^{p^{*}-\epsilon_{n}}+\frac{\left|u_{n}\right|^{*}(s)-\epsilon_{n}}{|x|^{s}}+a\left|u_{n}\right|^{p}\right) \phi^{p} d x .
$$

Then we have

$$
\int_{\mathcal{A}_{n}^{2}}\left|\nabla u_{n}\right|^{p} \phi^{p} d x \leq C \int_{\mathcal{A}_{n}^{2}}|\nabla \phi|^{p}|u|_{n}^{p} d x+C \int_{\mathcal{A}_{n}^{2}}\left(\left|u_{n}\right|^{p^{*}}+\frac{\left|u_{n}\right|^{p^{*}(s)}}{|x|^{s}}+\left|u_{n}\right|^{p}\right) \phi^{p} d x .
$$

(3.6) follows easily from above inequality.

Let $q>p^{*}(s)$. By Proposition 3.1, we have

$$
\begin{aligned}
\int_{\mathcal{A}_{n}^{2}} \frac{\phi^{p}\left|u_{n}\right|^{p^{*}(s)}}{|x|^{s}} d x & \leq\left(\int_{\mathcal{A}_{n}^{2}} \phi^{p}\left|u_{n}\right|^{q} d x\right)^{\frac{p^{*}(s)}{q}}\left(\int_{\mathcal{A}_{n}^{2}} \phi^{p}|x|^{-\frac{s q}{q-p^{*}(s)}} d x\right)^{\frac{q-p^{*}(s)}{q}} \\
& \leq C \lambda_{n}^{-\frac{p^{*}(s) N}{p q}} \lambda_{n}^{-\frac{1}{p}\left(N-\frac{s q}{q-p^{*}(s)}\right)\left(\frac{q-p^{*}(s)}{q}\right)}=C \lambda_{n}^{\frac{s-N}{p}}
\end{aligned}
$$

Now from (3.8), (3.6) and Proposition 3.1, we obtain that

$$
\int_{\mathcal{A}_{n}^{3}}\left|\nabla u_{n}\right|^{p} d x \leq C \lambda_{n}^{-\frac{N}{p}}+C \lambda_{n}^{\frac{p-N}{p}}+C \lambda_{n}^{\frac{s-N}{p}} \leq C \lambda_{n}^{\frac{p-N}{p}} .
$$

This proves (3.7). The proof of Proposition 3.3 is complete. 


\section{Proofs of main results}

In this section we prove Theorem 1.1 and Theorem 1.2. For notational simplicity, we write $p_{n}=p^{*}-\epsilon_{n}$ and $p_{n}(s)=p^{*}(s)-\epsilon_{n}$. Choose $t_{n} \in[\bar{C}+2, \bar{C}+3]$ such that

$$
\begin{aligned}
& \int_{\partial B}{ }_{t_{n} \lambda_{n}^{-\frac{1}{p}}\left(x_{n}\right)}\left(\mu\left|u_{n}\right|^{p_{n}}+\left|u_{n}\right|^{p}+\lambda_{n}^{-1}\left|\nabla u_{n}\right|^{p}+\lambda_{n}^{-\frac{s}{p}} \frac{\left|u_{n}\right|^{p_{n}(s)}}{|x|^{s}}\right) d \sigma \\
& \leq C \lambda_{n}^{\frac{1}{p}} \int_{\mathcal{A}_{n}^{3}}\left(\mu\left|u_{n}\right|^{p_{n}}+\left|u_{n}\right|^{p}+\lambda_{n}^{-1}\left|\nabla u_{n}\right|^{p}+\lambda_{n}^{-\frac{s}{p}} \frac{\left|u_{n}\right|^{p_{n}(s)}}{|x|^{s}}\right) d x \text {. }
\end{aligned}
$$

By Proposition 3.1, (3.7) and (3.8), we obtain that

$$
\int_{\partial B}\left(\mu\left|u_{n}\right|^{p_{n} \lambda_{n}^{-\frac{1}{p}}}+\left|u_{n}\right|^{p}+\lambda_{n}^{-1}\left|\nabla u_{n}\right|^{p}+\lambda_{n}^{-\frac{s}{p}} \frac{\left|u_{n}\right|^{p_{n}(s)}}{|x|^{s}}\right) d \sigma \leq C \lambda_{n}^{\frac{1-N}{p}} .
$$

We also have the following Pohozaev identity for $u_{n}$ on $B_{n}=B_{t_{n} \lambda_{n}^{-\frac{1}{p}}}\left(x_{n}\right) \cap \Omega$

$$
\begin{aligned}
& \left(\frac{N}{p_{n}}-\frac{N-p}{p}\right) \mu \int_{B_{n}}\left|u_{n}\right|^{p_{n}} d x+\int_{B_{n}}\left[a(x)-\frac{1}{p} \nabla a(x) \cdot\left(x-x_{0}\right)\right]\left|u_{n}\right|^{p} d x \\
& +\left(\frac{N-s}{p_{n}(s)}-\frac{N-p}{p}\right) \int_{B_{n}} \frac{\left|u_{n}\right|^{p_{n}(s)}}{|x|^{s}} d x+\frac{s}{p_{n}(s)} \int_{B_{n}} \frac{\left|u_{n}\right|^{p_{n}(s)}}{|x|^{2+s}}\left(x_{0} \cdot x\right) d x \\
& =\frac{N-p}{p} \int_{\partial B_{n}}\left|\nabla u_{n}\right|^{p-2} \frac{\partial u_{n}}{\partial \nu} u_{n} d \sigma+\int_{\partial B_{n}}\left|\nabla u_{n}\right|^{p-2} \nabla u_{n} \cdot\left(x-x_{0}\right) \frac{\partial u_{n}}{\partial \nu} d \sigma \\
& \quad-\frac{1}{p} \int_{\partial B_{n}}\left|\nabla u_{n}\right|^{p}\left(x-x_{0}\right) \cdot \nu d \sigma \\
& \quad+\int_{\partial B_{n}}\left(\frac{1}{p_{n}}\left|u_{n}\right|^{p_{n}}+\frac{1}{p_{n}(s)} \frac{\left|u_{n}\right|^{p_{n}(s)}}{|x|^{s}}+\frac{1}{p} a(x)\left|u_{n}\right|^{p}\right)\left(x-x_{0}\right) \cdot \nu d \sigma,
\end{aligned}
$$

where $\nu$ is the outward unit normal to $\partial B_{n}$ and $x_{0} \in \mathbf{R}^{N}$. Since $p_{n}<p^{*}$ and $p_{n}(s)<p^{*}(s)$, we have $\frac{N}{p_{n}}-\frac{N-p}{p}>0$ and $\frac{N-s}{p_{n}(s)}-\frac{N-p}{p}>0$. Thus we deduce from above the following inequality that

$$
\begin{aligned}
& \int_{B_{n}}\left[a(x)-\frac{1}{p} \nabla a(x) \cdot\left(x-x_{0}\right)\right]\left|u_{n}\right|^{p} d x+\frac{s}{p_{n}(s)} \int_{B_{n}} \frac{\left|u_{n}\right|^{p_{n}(s)}}{|x|^{2+s}}\left(x_{0} \cdot x\right) d x \\
& \leq \frac{N-p}{p} \int_{\partial B_{n}}\left|\nabla u_{n}\right|^{p-2} \frac{\partial u_{n}}{\partial \nu} u_{n} d \sigma+\int_{\partial B_{n}}\left|\nabla u_{n}\right|^{p-2} \nabla u_{n} \cdot\left(x-x_{0}\right) \frac{\partial u_{n}}{\partial \nu} d \sigma \\
& \quad-\frac{1}{p} \int_{\partial B_{n}}\left|\nabla u_{n}\right|^{p}\left(x-x_{0}\right) \cdot \nu d \sigma \\
& \quad+\int_{\partial B_{n}}\left(\frac{1}{p_{n}}\left|u_{n}\right|^{p_{n}}+\frac{1}{p_{n}(s)} \frac{\left|u_{n}\right|^{p_{n}(s)}}{|x|^{s}}+\frac{1}{p} a(x)\left|u_{n}\right|^{p}\right)\left(x-x_{0}\right) \cdot \nu d \sigma .
\end{aligned}
$$

Now we can prove Theorem 1.1.

Proof of Theorem 1.1. Since $\left\{x_{n}\right\} \subset \Omega$ is a bounded sequence, we may assume that $x_{n} \rightarrow x^{*} \in \bar{\Omega}$ as $n \rightarrow \infty$. We have two cases:

Case 1. $x^{*}=0$;

Case 2. $x^{*} \neq 0$. 
In Case 1 , choose $x_{0}=0$ in (4.3). Then we obtain that

$$
\begin{aligned}
& \int_{B_{n}}\left[a(x)-\frac{1}{p} \nabla a(x) \cdot x\right]\left|u_{n}\right|^{p} d x \\
& \leq \frac{N-p}{p} \int_{\partial B_{n}}\left|\nabla u_{n}\right|^{p-2} \frac{\partial u_{n}}{\partial \nu} u_{n} d \sigma+\int_{\partial B_{n}}\left|\nabla u_{n}\right|^{p-2} \nabla u_{n} \cdot x \frac{\partial u_{n}}{\partial \nu} d \sigma \\
& \quad-\frac{1}{p} \int_{\partial B_{n}}\left|\nabla u_{n}\right|^{p} x \cdot \nu d \sigma \\
& \quad+\int_{\partial B_{n}}\left(\frac{1}{p_{n}}\left|u_{n}\right|^{p_{n}}+\frac{1}{p_{n}(s)} \frac{\left|u_{n}\right|^{p_{n}(s)}}{|x|^{s}}+\frac{1}{p} a(x)\left|u_{n}\right|^{p}\right) x \cdot \nu d \sigma .
\end{aligned}
$$

Decompose $\partial B_{n}$ into $\partial B_{n}=\partial_{i} B_{n} \cup \partial_{e} B_{n}$, where $\partial_{i} B_{n}=\partial B_{n} \cap \Omega$ and $\partial_{e} B_{n}=$ $\partial B_{n} \cap \partial \Omega$. Consider the case $0 \in \partial \Omega$. Note that $u_{n}=0$ on $\partial \Omega$. Thus (4.4) implies that

$$
\begin{aligned}
L_{1}:= & \int_{B_{n}}\left[a(x)-\frac{1}{p} \nabla a(x) \cdot x\right]\left|u_{n}\right|^{p} d x-\left(1-\frac{1}{p}\right) \int_{\partial_{e} B_{n}}\left|\nabla u_{n}\right|^{p} x \cdot \nu d \sigma \\
\leq & \frac{N-p}{p} \int_{\partial_{i} B_{n}}\left|\nabla u_{n}\right|^{p-2} \frac{\partial u_{n}}{\partial \nu} u_{n} d \sigma+\int_{\partial_{i} B_{n}}\left|\nabla u_{n}\right|^{p-2} \nabla u_{n} \cdot x \frac{\partial u_{n}}{\partial \nu} d \sigma \\
& -\frac{1}{p} \int_{\partial_{i} B_{n}}\left|\nabla u_{n}\right|^{p} x \cdot \nu d \sigma \\
& +\int_{\partial_{i} B_{n}}\left(\frac{1}{p_{n}}\left|u_{n}\right|^{p_{n}}+\frac{1}{p_{n}(s)} \frac{\left|u_{n}\right|^{p_{n}(s)}}{|x|^{s}}+\frac{1}{p} a(x)\left|u_{n}\right|^{p}\right) x \cdot \nu d \sigma=: R_{1} .
\end{aligned}
$$

By assumption (1.9), we have that

$$
\int_{\partial_{e} B_{n}}\left|\nabla u_{n}\right|^{p} x \cdot \nu d \sigma \leq 0
$$

Recall that we assume $a(0)>0$. Thus (4.5) gives us

$$
L_{1} \geq \frac{1}{2} a(0) \int_{B_{n}}\left|u_{n}\right|^{p} d x
$$

On the other hand, since $|x| \leq C \lambda_{n}^{-1 / p}$ for $x \in \partial B_{n}$, we have by (4.2) that

$$
\begin{aligned}
R_{1} \leq & C \lambda_{n}^{-\frac{1}{p}} \int_{\partial_{i} B_{n}}\left(\left|u_{n}\right|^{p_{n}}+\left|u_{n}\right|^{p}+\left|\nabla u_{n}\right|^{p}+\frac{\left|u_{n}\right|^{p_{n}(s)}}{|x|^{s}}\right) d \sigma \\
& +C \int_{\partial_{i} B_{n}}\left|\nabla u_{n}\right|^{p-1}\left|u_{n}\right| d \sigma \leq C \lambda_{n}^{\frac{p-N}{p}} .
\end{aligned}
$$

Thus combining (4.5), (4.6) and (4.7) yields that

$$
\int_{B_{n}}\left|u_{n}\right|^{p} d x \leq C \lambda_{n}^{\frac{p-N}{p}} .
$$

Now we can follow the argument of [6] to obtain that

$$
\int_{B_{n}}\left|u_{n}\right|^{p} d x \geq C^{\prime} \lambda_{n}^{-p} .
$$

Therefore we arrive at

$$
\lambda_{n}^{-p} \leq C \lambda_{n}^{\frac{p-N}{p}} .
$$


Since $\lambda_{n} \rightarrow \infty$, (4.9) can not happen under the assumption that

$$
N>p^{2}+p \text {. }
$$

Now we consider the case $0 \in \Omega$. Then $\partial_{e} B_{n}=\emptyset$. So (4.5) holds with $\int_{\partial_{e} B_{n}}\left|\nabla u_{n}\right|^{p} x$. $\nu d \sigma=0$. By the same argument, we obtain (4.9). We reach a contradiction. $\emptyset$.

In Case 2, we have two possibilities: $B_{t_{n} \lambda_{n}^{-\frac{1}{p}}}\left(x_{n}\right) \subset \subset \Omega$ or $B_{t_{n} \lambda_{n}^{-\frac{1}{p}}}\left(x_{n}\right) \cap\left(\mathbf{R}^{N} \backslash \Omega\right) \neq$

Suppose that $B_{t_{n} \lambda_{n}^{-\frac{1}{p}}}\left(x_{n}\right) \subset \subset \Omega$. Then $B_{n}=B_{t_{n} \lambda_{n}^{-\frac{1}{p}}}\left(x_{n}\right)$. Take $x_{0}=x_{n}$ in (4.3). We obtain that

$$
\begin{aligned}
L_{2}:= & \frac{s}{p_{n}(s)} \int_{B_{n}} \frac{\left|u_{n}\right|^{p_{n}(s)}}{|x|^{2+s}}\left(x_{n} \cdot x\right) d x \\
\leq & -\int_{B_{n}}\left[a(x)-\frac{1}{p} \nabla a(x) \cdot\left(x-x_{n}\right)\right]\left|u_{n}\right|^{p} d x \\
& +\frac{N-p}{p} \int_{\partial B_{n}}\left|\nabla u_{n}\right|^{p-2} \frac{\partial u_{n}}{\partial \nu} u_{n} d \sigma+\int_{\partial B_{n}}\left|\nabla u_{n}\right|^{p-2} \nabla u_{n} \cdot\left(x-x_{n}\right) \frac{\partial u_{n}}{\partial \nu} d \sigma \\
& -\frac{1}{p} \int_{\partial B_{n}}\left|\nabla u_{n}\right|^{p}\left(x-x_{n}\right) \cdot \nu d \sigma \\
& +\int_{\partial B_{n}}\left(\frac{1}{p_{n}}\left|u_{n}\right|^{p_{n}}+\frac{1}{p_{n}(s)} \frac{\left|u_{n}\right|^{p_{n}(s)}}{|x|^{s}}+\frac{1}{p} a(x)\left|u_{n}\right|^{p}\right)\left(x-x_{n}\right) \cdot \nu d \sigma=: R_{2} .
\end{aligned}
$$

Since $x_{n} \rightarrow x^{*}$, we have $x_{n} \cdot x \geq \frac{1}{2}\left|x_{n}\right|^{2} \geq \frac{1}{4}\left|x^{*}\right|^{2}>0$ for $x \in B_{n}$. Thus

$$
L_{2} \geq C \int_{B_{n}}\left|u_{n}\right|^{p_{n}(s)} d x .
$$

Again, applying the same argument as that of [6] gives us that

$$
L_{2} \geq C \int_{B_{n}}\left|u_{n}\right|^{p_{n}(s)} d x \geq C^{\prime} \lambda_{n}^{-N+p_{n}(s) \frac{N-p}{p}} .
$$

On the other hand, by the same argument as that of (4.7), we obtain that

$$
R_{2} \leq C \lambda_{n}^{\frac{p-N}{p}}+C \int_{B_{n}}\left|u_{n}\right|^{p} d x
$$

in which the assumption $a \in C^{1}(\bar{\Omega})$ was used. We claim that

$$
\int_{B_{n}}\left|u_{n}\right|^{p} d x \leq C \lambda_{n}^{-p}
$$

Indeed, let $p_{1}>p^{*}$ such that $\frac{N}{p}\left(1-\frac{p}{p_{1}}\right)>p$. This is possible since $N>p^{2}+p$. Also, let $p_{2}=p$. Then we have $p^{*} / p^{\prime}<p_{2}<p^{*}$. By Proposition 2.1, there exist $v_{i} \geq 0, i=1,2$, such that $\left|u_{n}\right| \leq v_{1}+v_{2}$ and

$$
\left\|v_{1}\right\|_{*, p_{1}} \leq C, \quad\left\|v_{2}\right\|_{*, p} \leq C \lambda_{n}^{\frac{N-p}{p}-\frac{N}{p}}=C \lambda_{n}^{-1} .
$$

Hence by Hölder's inequality, we deduce that

$$
\int_{B_{n}}\left|u_{n}\right|^{p} d x \leq 2^{p-1} \int_{B_{n}}\left|v_{1}\right|^{p} d x+2^{p-1} \int_{B_{n}}\left|v_{2}\right|^{p} d x \leq C \lambda_{n}^{-\frac{N}{p}\left(1-\frac{p}{p_{1}}\right)}+C \lambda_{n}^{-p} \leq C \lambda_{n}^{-p} .
$$

This gives (4.13). Now combining (4.11)-(4.13) yields

$$
\lambda_{n}^{-N+\frac{N-p}{p} p_{n}(s)} \leq C \lambda_{n}^{-p}+C \lambda_{n}^{\frac{p-N}{p}} \leq C \lambda_{n}^{-p},
$$


since $N>p^{2}+p$. That is,

$$
\lambda_{n}^{p-s-\frac{N-p}{p} \epsilon_{n}} \leq C,
$$

which is impossible since $s<p$. We reach a contradiction.

It remains to consider $B_{t_{n} \lambda_{n}^{-\frac{1}{p}}}\left(x_{n}\right) \cap\left(\mathbf{R}^{N} \backslash \Omega\right) \neq \emptyset$. In this case, we take $x_{0} \in \mathbf{R}^{N} \backslash \Omega$ in (4.3) such that $\left|x_{0}-x_{n}\right| \leq 2 t_{n} \lambda_{n}^{-\frac{1}{p}}$ and $\nu \cdot\left(x-x_{0}\right) \leq 0$ on $\partial \Omega \cap B_{n}$. With this $x_{0}$, we get from (4.3),

$$
\begin{aligned}
& \frac{s}{p_{n}(s)} \int_{B_{n}} \frac{\left|u_{n}\right|^{p_{n}(s)}}{|x|^{2+s}}\left(x_{n} \cdot x\right) d x \\
& \leq-\int_{B_{n}}\left[a(x)-\frac{1}{p} \nabla a(x) \cdot\left(x-x_{n}\right)\right]\left|u_{n}\right|^{p} d x \\
& \quad+\frac{N-p}{p} \int_{\partial_{i} B_{n}}\left|\nabla u_{n}\right|^{p-2} \frac{\partial u_{n}}{\partial \nu} u_{n} d \sigma+\int_{\partial_{i} B_{n}}\left|\nabla u_{n}\right|^{p-2} \nabla u_{n} \cdot\left(x-x_{n}\right) \frac{\partial u_{n}}{\partial \nu} d \sigma \\
& \quad-\frac{1}{p} \int_{\partial_{i} B_{n}}\left|\nabla u_{n}\right|^{p}\left(x-x_{n}\right) \cdot \nu d \sigma \\
& \quad+\int_{\partial_{i} B_{n}}\left(\frac{1}{p_{n}}\left|u_{n}\right|^{p_{n}}+\frac{1}{p_{n}(s)} \frac{\left|u_{n}\right|^{p_{n}(s)}}{|x|^{s}}+\frac{1}{p} a(x)\left|u_{n}\right|^{p}\right)\left(x-x_{n}\right) \cdot \nu d \sigma .
\end{aligned}
$$

Arguing as above, we find that (4.14) still holds. Thus we obtain a contradiction. The proof of Theorem 1.1 is complete.

Now we can prove Theorem 1.2.

Proof of Theorem 1.2. The proof is standard. We give a sketch of proof for the readers' convenience, and refer to Cao, Peng and Yan [6] for more details. First we prove that for each $k \geq 1$, there exists a bounded sequence of $\left\{u_{k, \epsilon_{n}}\right\}_{n} \subset W_{0}^{1, p}(\Omega)$ and $\left\{c_{k, \epsilon_{n}}\right\} \subset \mathbf{R}$ such that $I_{\epsilon_{n}}^{\prime}\left(u_{k, \epsilon_{n}}\right)=0, I_{\epsilon_{n}}\left(u_{k, \epsilon_{n}}\right)=c_{k, \epsilon_{n}}$ and $c_{k, \epsilon_{n}} \rightarrow c_{k} \in \mathbf{R}$ as $n \rightarrow \infty$. Indeed, this follows from a standard min-max argument as below (see e.g. Ghoussoub [17]).

For each $k$, define the $\mathbf{Z}_{2}$-homotopy class $\mathcal{F}_{k}$ by

$$
\mathcal{F}_{k}=\left\{A: A \subset W_{0}^{1, p}(\Omega) \text { is compact, } \mathbf{Z}_{2} \text {-invariant, and } \gamma(A) \geq k\right\},
$$

where the genus $\gamma(A)$ is the smallest integer $m$, such that there exists an odd continuous mapping $\phi \in C\left(A, \mathbf{R}^{m} \backslash\{0\}\right)$, and then define the min-max value (see property (I3) in page 134 of Ghoussoub [17])

$$
c_{k, \epsilon}=\min _{A \in \mathcal{F}_{k}} \max _{u \in A} I_{\epsilon}(u)
$$

for all $\epsilon>0$. Since $I_{\epsilon}$ is an even functional that satisfies the Palais-Smale condition in all energy levels, we use Corollary 7.12 of Ghoussoub [17] to conclude that $c_{k, \epsilon}$ is a critical value of $I_{\epsilon}$. Thus, there exists $u_{k, \epsilon}$ such that $I_{\epsilon}\left(u_{k, \epsilon}\right)=c_{k, \epsilon}$. By the same argument as in Cao, Peng and Yan [6], we can deduce that $c_{k, \epsilon}$ is bounded uniformly for $\epsilon$ small, and that $\left\{u_{k, \epsilon}\right\} \subset W_{0}^{1, p}(\Omega)$ is a bounded sequence as $\epsilon \rightarrow 0$. Hence we can apply Theorem 1.1 to infer that (up to a subsequence) $u_{k, \epsilon_{n}} \rightarrow u_{k}$ strongly in $W_{0}^{1, p}(\Omega)$ as $n \rightarrow \infty$, and $I^{\prime}\left(u_{k}\right)=0$ with $I\left(u_{k}\right)=c_{k}$.

Thus we get a sequence $\left\{c_{k}\right\}_{k}$ of critical values of $I$. Now, two cases may occur:

Case I: the set $\left\{c_{k}\right\}_{k}$ is infinite. In this case, it is obvious that $I$ has infinitely many different critical points, and thus Theorem 1.2 holds;

Case II: for some $m \geq 1$, we have $c_{k}=c$ for all $k \geq m$. 
We have to prove that Theorem 1.2 holds in the latter case as well. With no loss of generality, assume that $c$ is an isolated critical value of $I$ in the sense that, for some $\delta_{0}>0, I$ has no critical value in $\left(c-\delta_{0}, c+\delta_{0}\right) \backslash\{c\}$. Fix such $\delta_{0}$. Denote

$$
K_{c}=\left\{u \in W_{0}^{1, p}(\Omega): I(u)=c, I^{\prime}(u)=0\right\} .
$$

Note that $K_{c}$ is $\mathbf{Z}_{2}$-invariant since $I$ is an odd functional, and $K_{c}$ is also compact due to Theorem 1.1. The idea is to prove that $K_{c}$ is an infinite set, from which Theorem 1.2 follows. Since any finite $\mathbf{Z}_{2}$-invariant set has genus one, it suffices to prove

$$
\gamma\left(K_{c}\right) \geq 2
$$

We argue by contradiction. Suppose, on the contrary, that $\gamma\left(K_{c}\right)=1$. Denote

$$
\mathcal{K}=\left\{u \in W_{0}^{1, p}(\Omega):\left\|u-K_{c}\right\| \leq \delta_{1}\right\},
$$

where $0<\delta_{1}<\delta_{0}$ is so small that $\gamma(\mathcal{K})=1$. Such $\delta_{1}$ exists due to the fact that $K_{c}$ is compact and $\mathbf{Z}_{2}$-invariant (see page 132 of Ghoussoub [17]). For each $\epsilon>0$, define

$$
D_{\epsilon}=\left(I_{\epsilon}^{c+\delta_{1}} \backslash I_{\epsilon}^{c-\delta_{1}}\right) \backslash \mathcal{K},
$$

where $I_{\epsilon}^{t}$ denotes the level set of $I_{\epsilon}$ given by

$$
I_{\epsilon}^{t}=\left\{u \in W_{0}^{1, p}(\Omega): I_{\epsilon}(u)<t\right\} .
$$

We claim that $I_{\epsilon}$ has no critical point in $D_{\epsilon}$ for $\epsilon$ sufficiently small. Otherwise, there exist $\epsilon_{n} \rightarrow 0$ and $u_{n} \in D_{\epsilon_{n}}$ such that $I_{\epsilon_{n}}^{\prime}\left(u_{n}\right)=0$ and $I_{\epsilon_{n}}\left(u_{n}\right) \in\left[c-\delta_{1}, c+\delta_{1}\right)$. Then, applying Theorem 1.1 yields that (up to a subsequence) $u_{n}$ converges to $u$ strongly in $W_{0}^{1, p}(\Omega)$ as $n \rightarrow \infty$. Then we have $I^{\prime}(u)=0$ and $I(u) \in\left(c-\delta_{0}, c+\delta_{0}\right)$. Thus $u \in K_{c} \subset \mathcal{K}$ holds since $c$ is assumed to be an isolated critical value of $I$. However, note also that $u_{n} \notin \mathcal{K}$ implies $u \notin \mathcal{K}$. We reach a contradiction. Hence, we conclude that for every $\epsilon>0$ sufficiently small, there exists $c_{\epsilon}^{*}$ such that

$$
\left\|I_{\epsilon}^{\prime}(u)\right\| \geq c_{\epsilon}^{*}>0 \quad \text { for } u \in D_{\epsilon} .
$$

With the help of above lower bound, standard deformation techniques (pseudogradient flow) yield an odd homeomorphism $\eta: W_{0}^{1, p}(\Omega) \rightarrow W_{0}^{1, p}(\Omega)$ such that

$$
\eta\left(I_{\epsilon}^{c+\delta_{1}} \backslash \mathcal{K}\right) \subset I_{\epsilon}^{c-\delta_{1}} .
$$

See for example the proof of Theorem 1.9 of Rabinowitz [27]. Note that we need to replace the modified pseudo-gradient vector field $V$ defined in page 150 of Rabinowitz [27] by $V / c_{\epsilon}^{*}$.

Now we are ready to prove (4.15). Fix $k>m$. Since $c_{k, \epsilon}$ and $c_{k+1, \epsilon} \rightarrow c$ as $\epsilon \rightarrow 0$, we have

$$
c_{k, \epsilon}, c_{k+1, \epsilon} \in\left(c-\delta_{1} / 4, c+\delta_{1} / 4\right)
$$

for $\epsilon$ sufficiently small. By the definition of $c_{k+1, \epsilon}$, there exists $A \in \mathcal{F}_{k+1}$ such that

$$
\max _{A} I_{\epsilon}<c_{k+1, \epsilon}+\delta_{1} / 4<c+\delta_{1}
$$

which implies that $A \subset I_{\epsilon}^{c+\delta_{1}}$. Then $\tilde{A}:=\eta(A \backslash \mathcal{K}) \subset I_{\epsilon}^{c-\delta_{1}}$ holds. That is,

$$
I_{\epsilon}(u)<c-\delta_{1} \quad \text { for } u \in \tilde{A} .
$$

We claim that $\tilde{A} \in \mathcal{F}_{k}$. Indeed, since $\gamma$ is subadditive, we deduce that $\gamma(A \backslash \mathcal{K}) \geq$ $\gamma(A)-\gamma(\mathcal{K}) \geq k$ since we assume $\gamma(\mathcal{K})=1$. Thus $A \backslash \mathcal{K} \subset \mathcal{F}_{k}$ holds. Hence the 
supervariant of $\gamma$ implies that $\tilde{A} \in \mathcal{F}_{k}$ (see Theorem 1.9 of Rabinowitz [27]). As a result,

$$
c_{k, \epsilon} \leq \sup _{\tilde{A}} I_{\epsilon} \leq c-\delta_{1} .
$$

This contradicts to $c_{k, \epsilon} \geq c-\delta_{1} / 4$. Hence $\gamma\left(K_{c}\right) \geq 2$ and the proof of Theorem 1.2 is complete.

Acknowledgments. Both authors would like to thank Xiao Zhong for many useful discussions. They would also like to thank the anonymous referees for valuable comments which helped to improve the manuscript.

\section{Appendix A. Estimates for quasilinear problems with Hardy potential}

In this section, we deduce some elementary estimates for solutions of a quasilinear elliptic problem involving a Hardy potential. Let $D$ be a bounded domain in $\mathbf{R}^{N}$ and $0 \in D$. For any $0 \leq t<p$, write $d \mu_{t}=|x|^{-t} d x$ and $\|w\|_{q, \mu_{t}}^{q}=\int_{D}|w|^{q} d \mu_{t}$. We also use the notation $\|w\|_{q}=\|w\|_{q, \mu_{0}}$. Let us recall that

$$
\|w\|_{*, q}=\|w\|_{q}+\|w\|_{\frac{(N-s)}{N} q, \mu_{s}} .
$$

Proposition A.1. For any $f_{i} \geq 0$ and $f_{i} \in L^{\infty}(D), i=1,2$, let $w \in W_{0}^{1, p}(D)$ be the solution of

$$
\begin{cases}-\Delta_{p} w=f_{1}(x)+\frac{f_{2}(x)}{|x|^{s}} & x \in D, \\ w=0 & \text { on } \partial D .\end{cases}
$$

Then, for any $1<q<N / p$, there exists $C=C(N, p, s, q)>0$ such that

$$
\|w\|_{*, \frac{(p-1) N q}{N-p q}} \leq C\left(\left\|f_{1}\right\|_{q}+\left\|f_{2}\right\|_{\frac{(N-s) q}{N-s q}, \mu_{s}}\right)^{\frac{1}{p-1}} .
$$

Proof. By the maximum principle, we find that $w \geq 0$. We claim that if $r>1 / p^{\prime}$, then

$$
\|w\|_{*, p^{*} r}^{p r} \leq C \int_{D}\left(f_{1}+\frac{f_{2}}{|x|^{s}}\right) w^{1+p(r-1)} d x
$$

for $C=C(N, p, s, r)>0$.

First, we consider the case when $r \geq 1$. Since $f_{1}, f_{2}$ are bounded functions, it is standard to prove that $w \in L^{\infty}(D)$ by Moser's iteration method [25]. Then we can take a test function $\xi=w^{1+p(r-1)}$ so that

$$
\frac{1+p(r-1)}{r^{p}} \int_{D}\left|\nabla w^{r}\right|^{p} d x=\int_{D}\left(f_{1}+\frac{f_{2}}{|x|^{s}}\right) w^{1+p(r-1)} d x
$$

Applying the Sobolev inequality and the Caffarelli-Kohn-Nirenberg inequality gives us

$$
\|w\|_{p^{*} r}^{p r}+\|w\|_{p^{*}(s) r, \mu_{s}}^{p r} \leq C \int_{D}\left|\nabla w^{r}\right|^{p} d x
$$

for $C=C(N, p, s, r)>0$. That is,

$$
\|w\|_{*, p^{*} r}^{p r} \leq C \int_{D}\left|\nabla w^{r}\right|^{p} d x
$$

Then, combining (A.2) and (A.3) yields (A.1).

Next, consider the case when $1 / p^{\prime}<r<1$. Let $\epsilon>0$. Define $\xi=w(w+\epsilon)^{p(r-1)}$. It is direct to verify that $\xi \in W_{0}^{1, p}(D)$ and

$$
\nabla \xi=(w+\epsilon)^{p(r-1)} \nabla w+p(r-1) w(w+\epsilon)^{p(r-1)-1} \nabla w .
$$


Take $\xi$ as a test function. We have

$$
\int_{D}|\nabla w|^{p-2} \nabla w \cdot \nabla \xi d x=\int_{D}\left(f_{1}+\frac{f_{2}}{|x|^{s}}\right) \xi d x
$$

A direct calculation gives that

$$
\begin{aligned}
\int_{D}|\nabla w|^{p-2} \nabla w \cdot \nabla \xi d x & \geq(1+p(r-1)) \int_{D}(w+\epsilon)^{p(r-1)}|\nabla w|^{p} d x \\
& =\frac{1+p(r-1)}{r^{p}} \int_{D}\left|\nabla\left((w+\epsilon)^{r}-\epsilon^{r}\right)\right|^{p} d x \\
& \geq C\left(\left\|\left((w+\epsilon)^{r}-\epsilon^{r}\right)\right\|_{p^{*}}^{p}+\left\|\left((w+\epsilon)^{r}-\epsilon^{r}\right)\right\|_{p^{*}(s), \mu_{s}}^{p}\right),
\end{aligned}
$$

for $C=C(N, p, s, r)>0$.

Write $w_{\epsilon}=\left((w+\epsilon)^{r}-\epsilon^{r}\right)^{1 / r}$. Then there exists $C>0$ such that

$$
\int_{D}|\nabla w|^{p-2} \nabla w \cdot \nabla \xi d x \geq C\left\|w_{\epsilon}\right\|_{*, p^{*} r}^{p r}
$$

Thus

$$
\left\|w_{\epsilon}\right\|_{*, p^{*} r}^{p r} \leq C \int_{D}\left(f_{1}+\frac{f_{2}}{|x|^{s}}\right) w(w+\epsilon)^{p(r-1)} d x \leq C \int_{D}\left(f_{1}+\frac{f_{2}}{|x|^{s}}\right) w^{1+p(r-1)} d x .
$$

Letting $\epsilon \rightarrow 0$, we obtain (A.1) in the case when $r \in\left(1 / p^{\prime}, 1\right)$.

To prove Proposition A.1, we apply Hölder's inequality to (A.1) and obtain that

$$
\begin{aligned}
\|w\|_{*, p^{*} r}^{p r} & \leq C\left(\left\|f_{1}\right\|_{\frac{p^{*} r}{p^{*} r-(1+p(r-1))}}+\left\|f_{2}\right\|_{\frac{p^{*}(s) r}{p^{*}(s) r-(1+p(r-1))}, \mu_{s}}\right)\left(\|w\|_{p^{*} r}^{1+p(r-1)}+\|w\|_{p^{*}(s) r, \mu_{s}}^{1+p(r-1)}\right) \\
& \leq C\left(\left\|f_{1}\right\|_{\frac{p^{*} r}{p^{*} r-(1+p(r-1))}}+\left\|f_{2}\right\|_{\frac{p^{*}(s) r}{p^{*}(s) r-(1+p(r-1))}, \mu_{s}}\right)\|w\|_{*, p^{*} r}^{1+p(r-1)},
\end{aligned}
$$

which implies that

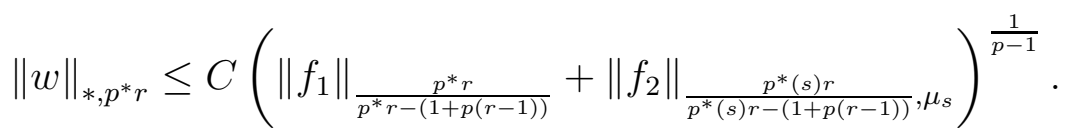

Now for any $q \in(1, N / p)$. Let $r \in\left(1 / p^{\prime}, \infty\right)$ be such that $q=\frac{p^{*} r}{p^{*} r-(1+p(r-1))}$. Then

$$
\frac{p^{*}(s) r}{p^{*}(s) r-(1+p(r-1))}=\frac{(N-s) q}{N-s q} \text { and } p^{*} r=\frac{(p-1) N q}{N-p q} \text {. }
$$

Hence

We finish the proof.

$$
\|w\|_{*, \frac{(p-1) N q}{N-p q}} \leq C\left(\left\|f_{1}\right\|_{q}+\left\|f_{2}\right\|_{\frac{(N-s) q}{N-s q}, \mu_{s}}\right) \frac{1}{p-1} .
$$

As an application of Proposition A.1 we have the following corollary.

Corollary A.2. Let $w \in W_{0}^{1, p}(D)$ be the solution of

$$
\begin{cases}-\Delta_{p} w=\left(a_{1}(x)+\frac{a_{2}(x)}{|x|^{s}}\right) v^{p-1} & x \in D \\ w=0 & \text { on } \partial D\end{cases}
$$

where $a_{1}, a_{2}, v \in L^{\infty}(D)$ are nonnegative functions. Then for any $\infty>q>p^{*} / p^{\prime}$, there holds

$$
\|w\|_{*, q} \leq C\left(\left\|a_{1}\right\|_{\frac{N}{p}}+\left\|a_{2}\right\|_{\frac{N-s}{p-s}, \mu_{s}}\right)^{\frac{1}{p-1}}\|v\|_{*, q}
$$


for $C=C(N, p, s, q)>0$.

Proof. Let $\infty>q>p^{*} / p^{\prime}$ and define $r=N q /(N(p-1)+p q)$. Then $1<r<N / p$ and $q=(p-1) N r /(N-p r)$.

By applying Proposition A.1 with $f_{i}=a_{i} v^{p-1}, i=1,2$, we obtain that

$$
\|w\|_{*, q} \leq C\left(\left\|f_{1}\right\|_{r}+\left\|f_{2}\right\|_{\frac{(N-s) r}{N-s r}, \mu_{s}}\right)^{\frac{1}{p-1}},
$$

for $C=C(N, p, s, q)>0$. By Hölder's inequality and the definition of $\|\cdot\|_{*, q}$, we have that

and that

$$
\left\|f_{1}\right\|_{r} \leq\left\|a_{1}\right\|_{\frac{N}{p}}\|v\|_{q}^{p-1} \leq\left\|a_{1}\right\|_{\frac{N}{p}}\|v\|_{*, q}^{p-1}
$$

$$
\left\|f_{2}\right\|_{\frac{(N-s) r}{N-s r}, \mu_{s}} \leq\left\|a_{2}\right\|_{\frac{N-s}{p-s}, \mu_{s}}\|v\|_{\frac{(N-s) q}{N}, \mu_{s}}^{p-1} \leq\left\|a_{2}\right\|_{\frac{N-s}{p-s}, \mu_{s}}\|v\|_{*, q}^{p-1} .
$$

Combining the above inequalities gives Corollary A.2.

We also have the following corollary.

Corollary A.3. Let $w \in W_{0}^{1, p}(D)$ be the solution of

$$
\begin{cases}-\Delta_{p} w=\left(a_{1}(x)+\frac{a_{2}(x)}{|x|^{s}}\right) v^{p-1} & x \in D, \\ w=0 & \text { on } \partial D,\end{cases}
$$

where $a_{1}, a_{2}, v \in L^{\infty}(D)$ are nonnegative functions. Then for any $p^{*}>p_{2}>p^{*} / p^{\prime}$, there holds

$$
\|w\|_{*, p_{2}} \leq C\left(\left\|a_{1}\right\|_{r_{1}}+\left\|a_{2}\right\|_{r_{2}, \mu_{s}}\right)^{\frac{1}{p-1}}\|v\|_{*, p^{*}}
$$

for $C=C\left(N, p, s, p_{2}\right)>0$, where $r_{1}, r_{2}$ are defined by

$$
\frac{1}{r_{1}}=(p-1)\left(\frac{1}{p_{2}}-\frac{1}{p^{*}}\right)+\frac{p}{N}
$$

and

$$
\frac{1}{r_{2}}=(p-1)\left(\frac{N}{(N-s) p_{2}}-\frac{1}{p^{*}(s)}\right)+\frac{p-s}{N-s} .
$$

Proof. The proof is similar to that of Corollary A.2. By applying Proposition A.1 with $f_{i}=a_{i} v^{p-1}, i=1,2$, we obtain

$$
\|w\|_{*, p_{2}} \leq C\left(\left\|f_{1}\right\|_{\frac{N p_{2}}{(p-1) N+p p_{2}}}+\left\|f_{2}\right\|_{\frac{(N-s) p_{2}}{(p-1) N+(p-s) p_{2}}, \mu_{s}}\right)^{\frac{1}{p-1}} .
$$

Let $r_{1}, r_{2}$ be defined as in (A.4) (A.5). Applying Hölder's inequality gives us that

$$
\left\|f_{1}\right\|_{\frac{N p_{2}}{(p-1) N+p p_{2}}} \leq\left\|a_{1}\right\|_{r_{1}}\|v\|_{p^{*}}^{p-1}
$$

and that

$$
\left\|f_{2}\right\|_{\frac{(N-s) p_{2}}{(p-1) N+(p-s) p_{2}}, \mu_{s}} \leq\left\|a_{2}\right\|_{r_{2}, \mu_{s}}\|v\|_{p^{*}(s), \mu_{s}}^{p-1} .
$$

Combining the above inequalities gives Corollary A.3.

In the end of this section, we give the following result.

Lemma A.4. Let $w \in W_{\text {loc }}^{1, p}\left(\mathbf{R}^{N}\right), w \geq 0$, be a weak solution of the equation

$$
-\Delta_{p} w \leq\left(a_{1}(x)+\frac{a_{2}(x)}{|x|^{s}}\right) w^{p-1}
$$


in $\mathbf{R}^{N}$, where $a_{1}, a_{2} \in L_{\text {loc }}^{\infty}\left(\mathbf{R}^{N}\right)$ are nonnegative functions. Then, for any unit ball $B_{1}(y) \subset \mathbf{R}^{N}$ and for any $q>p^{*}$, there is a small constant $\delta=\delta(q)>0$ such that if

$$
\left(\int_{B_{1}(y)} a_{1}^{\frac{N}{p}} d x\right)^{\frac{p}{N}}+\left(\int_{B_{1}(y)} a_{2}^{\frac{N-s}{p-s}} d \mu_{s}\right)^{\frac{p-s}{N-s}}<\delta
$$

then for any $\gamma \in\left(0, p^{*}\right)$, there holds

$$
\|w\|_{q, B_{1 / 2}(y)} \leq C\|w\|_{\gamma, B_{1}(y)}
$$

for $C=C(N, p, s, q, \gamma)>0$.

Proof. For simplicity, we write $B_{r}=B_{r}(y)$ for $r>0$ in the following proof. It is standard to show that $w \in L_{\text {loc }}^{\infty}\left(\mathbf{R}^{N}\right)$ by Moser's iteration method [25]. For any $\eta \in C_{0}^{\infty}\left(B_{1}\right)$, take $\varphi=\eta^{p} w^{1+p(\tau-1)}, \tau \geq 1$, as a test function. We have

$$
\int_{B_{1}}|\nabla w|^{p-2} \nabla w \cdot \nabla \varphi d x \leq \int_{B_{1}}\left(a_{1}(x)+\frac{a_{2}(x)}{|x|^{s}}\right) \eta^{p} w^{p \tau} d x .
$$

First, a simple calculation gives us that

$$
\int_{B_{1}}|\nabla w|^{p-2} \nabla w \cdot \nabla \varphi d x \geq C \int_{B_{1}}\left|\nabla\left(\eta w^{\tau}\right)\right|^{p} d x-C \int_{B_{1}}|\nabla \eta|^{p} w^{p \tau} d x
$$

for $C=C(N, p, \tau)>0$. Second, by Hölder's inequality, we have

$$
\int_{B_{1}} a_{1}(x) \eta^{p} w^{p \tau} d x \leq\left(\int_{B_{1}} a_{1}^{\frac{N}{p}} d x\right)^{\frac{p}{N}}\left(\int_{B_{1}}\left(\eta w^{\tau}\right)^{p^{*}} d x\right)^{\frac{p}{p^{*}}},
$$

and

$$
\int_{B_{1}} \frac{a_{2}(x)}{|x|^{s}} \eta^{p} w^{p \tau} d x \leq\left(\int_{B_{1}} a_{2}^{\frac{N-s}{p-s}} d \mu_{s}\right)^{\frac{p-s}{N-s}}\left(\int_{B_{1}}\left(\eta w^{\tau}\right)^{p^{*}(s)} d \mu_{s}\right)^{\frac{p}{p^{*}(s)}} .
$$

Thus (A.6) implies that

$$
\begin{aligned}
\int_{B_{1}}\left|\nabla\left(\eta w^{\tau}\right)\right|^{p} d x \leq & C \int_{B_{1}}|\nabla \eta|^{p} w^{p \tau} d x \\
& +C A\left(\left(\int_{B_{1}}\left(\eta w^{\tau}\right)^{p^{*}} d x\right)^{\frac{p}{p^{*}}}+\left(\int_{B_{1}}\left(\eta w^{\tau}\right)^{p^{*}(s)} d \mu_{s}\right)^{\frac{p}{p^{*}(s)}}\right),
\end{aligned}
$$

where $C=C(N, p, s, \tau)>0$ and

$$
A=\left(\int_{B_{1}} a_{1}^{\frac{N}{p}} d x\right)^{\frac{p}{N}}+\left(\int_{B_{1}} a_{2}^{\frac{N-s}{p-s}} d \mu_{s}\right)^{\frac{p-s}{N-s}} .
$$

By the Sobolev inequality and the Caffarelli-Kohn-Nirenberg inequality, we obtain that

$$
\left(\int_{B_{1}}\left(\eta w^{\tau}\right)^{p^{*}} d x\right)^{\frac{p}{p^{*}}}+\left(\int_{B_{1}}\left(\eta w^{\tau}\right)^{p^{*}(s)} d \mu_{s}\right)^{\frac{p}{p^{*}(s)}} \leq C(N, p, s) \int_{B_{1}}\left|\nabla\left(\eta w^{\tau}\right)\right|^{p} d x .
$$

Combining (A.7) and (A.8) yields that

$$
\begin{aligned}
& \left(\int_{B_{1}}\left(\eta w^{\tau}\right)^{p^{*}} d x\right)^{\frac{p}{p^{*}}}+\left(\int_{B_{1}}\left(\eta w^{\tau}\right)^{p^{*}(s)} d \mu_{s}\right)^{\frac{p}{p^{*}(s)}} \\
& \leq C \int_{B_{1}}|\nabla \eta|^{p} w^{p \tau} d x+C A\left(\left(\int_{B_{1}}\left(\eta w^{\tau}\right)^{p^{*}} d x\right)^{\frac{p}{p^{*}}}+\left(\int_{B_{1}}\left(\eta w^{\tau}\right)^{p^{*}(s)} d \mu_{s}\right)^{\frac{p}{p^{*}(s)}}\right) .
\end{aligned}
$$


Thus we can choose

$$
\delta=\delta(\tau)>0
$$

small enough such that if $A<\delta=\delta(\tau)$, then $C A<1 / 2$ and thus

$$
\left(\int_{B_{1}}\left(\eta w^{\tau}\right)^{p^{*}} d x\right)^{\frac{p}{p^{*}}}+\left(\int_{B_{1}}\left(\eta w^{\tau}\right)^{p^{*}(s)} d \mu_{s}\right)^{\frac{p}{p^{*}(s)}} \leq C \int_{B_{1}}|\nabla \eta|^{p} w^{p \tau} d x
$$

for $C=C(N, p, s, \tau)>0$. In particular, if $A<\delta$, we have

$$
\left(\int_{B_{1}}\left(\eta w^{\tau}\right)^{p^{*}} d x\right)^{\frac{p}{p^{*}}} \leq C \int_{B_{1}}|\nabla \eta|^{p} w^{p \tau} d x
$$

for $C=C(N, p, s, \tau)>0$.

Let $0<r<R \leq 1$ and $\eta \in C_{0}^{\infty}\left(B_{R}\right)$ be a cut-off function such that $0 \leq \eta \leq 1$, $\eta \equiv 1$ in $B_{r}$ and $|\nabla \eta| \leq 2 /(R-r)$. Substituting $\eta$ into (A.10) gives us that

$$
\left(\int_{B_{r}} w^{p \chi \tau} d x\right)^{\frac{1}{\chi}} \leq \frac{C(\tau)}{(R-r)^{p}} \int_{B_{R}} w^{p \tau} d x
$$

where $\chi=p^{*} / p>1$.

Now for any fixed $q>p^{*}$, there exists $k \in \mathbf{N}$ such that $p \chi^{k} \leq q<p \chi^{k+1}$. Let $\tau_{i}=\chi^{i}, r_{i}=r+(R-r) / 2^{i-1}, i=1, \ldots, k$, and let

$$
\delta=\min \left\{\delta\left(\tau_{1}\right), \cdots, \delta\left(\tau_{k}\right)\right\}
$$

where $\delta\left(\tau_{i}\right)$ is defined by (A.9) with $\tau=\tau_{i}, i=1, \cdots, k$. Then if $A<\delta$, we obtain by $($ A.11)

$$
\left(\int_{B_{r_{i+1}}} w^{p \chi^{i+1}} d x\right)^{\frac{1}{p \chi^{i+1}}} \leq \frac{C\left(\tau_{i}\right)}{\left(r_{i}-r_{i+1}\right)^{1 / \chi^{i}}}\left(\int_{B_{r_{i}}} w^{p \chi^{i}} d x\right)^{\frac{1}{p \chi^{i}}},
$$

for $i=1, \ldots, k$. Iterate the above inequality from $i=1$ to $i=k$. We obtain

$$
\left(\int_{B_{r_{k+1}}} w^{p \chi^{k+1}} d x\right)^{\frac{1}{p \chi^{k+1}}} \leq \frac{C}{\left(R-r_{k+1}\right)^{\sigma}}\left(\int_{B_{R}} w^{p^{*}} d x\right)^{\frac{1}{p^{*}}}
$$

for some constants $C>0$ and $\sigma>0$. As a result, we have

$$
\left(\int_{B_{r}} w^{q} d x\right)^{\frac{1}{q}} \leq \frac{C}{(R-r)^{\sigma}}\left(\int_{B_{R}} w^{p^{*}} d x\right)^{\frac{1}{p^{*}}},
$$

since $q<p \chi^{k+1}$.

Fix $\gamma \in\left(0, p^{*}\right)$. There exists $\theta \in(0,1)$ such that

$$
\frac{1}{p^{*}}=\frac{\theta}{\gamma}+\frac{1-\theta}{q}
$$

Thus by Hölder's inequality and Young's inequality, (A.12) implies that

$$
\left(\int_{B_{r}} w^{q} d x\right)^{\frac{1}{q}} \leq \frac{1}{2}\left(\int_{B_{R}} w^{q} d x\right)^{\frac{1}{q}}+\frac{C}{(R-r)^{\sigma / \theta}}\left(\int_{B_{R}} w^{\gamma} d x\right)^{\frac{1}{\gamma}} .
$$

In particular, there holds

$$
\left(\int_{B_{r}} w^{q} d x\right)^{\frac{1}{q}} \leq \frac{1}{2}\left(\int_{B_{R}} w^{q} d x\right)^{\frac{1}{q}}+\frac{C}{(R-r)^{\sigma / \theta}}\left(\int_{B_{1}} w^{\gamma} d x\right)^{\frac{1}{\gamma}} .
$$


Now Applying Lemma 4.3 of Han and Lin [22, Chapter 4] (see Lemma A.5 below) yields

$$
\left(\int_{B_{r}} w^{q} d x\right)^{\frac{1}{q}} \leq \frac{C}{(R-r)^{\sigma / \theta}}\left(\int_{B_{1}} w^{\gamma} d x\right)^{\frac{1}{\gamma}}
$$

for some constants $C>0$. Choose $r=1 / 2$ and $R=1$. We complete the proof.

We attach Lemma 4.3 of Han and Lin [22, Chapter 4] here for the readers' convenience.

Lemma A.5. Let $f \geq 0$ be a bounded function in $\left[\tau_{0}, \tau_{1}\right]$ with $\tau_{0} \geq 0$. Suppose for $\tau_{0} \leq t<s \leq \tau_{1}$ we have

$$
f(t) \leq \theta f(s)+\frac{A}{(s-t)^{\alpha}}+B
$$

for some $\theta \in(0,1]$ and some nonnegative constants $A, B, \alpha$. Then, there exists $c(\alpha, \theta)>0$ such that, for any $\tau_{0} \leq t<s \leq \tau_{1}$, there holds

$$
f(t) \leq c(\alpha, \theta)\left\{\frac{A}{(s-t)^{\alpha}}+B\right\} .
$$

\section{Appendix B. A decay estimate}

We use $\mathbf{R}_{*}^{N}$ to denote either $\mathbf{R}^{N}$ or $\mathbf{R}_{+}^{N}$. Consider the following equation

$$
\left\{\begin{array}{l}
-\Delta_{p} u=\mu|u|^{p^{*}-2} u+\frac{|u|^{p^{*}(s)-2} u}{|x|^{s}} \text { in } \mathbf{R}_{*}^{N}, \\
u \in \mathcal{D}_{0}^{1, p}\left(\mathbf{R}_{*}^{N}\right),
\end{array}\right.
$$

where $\mathcal{D}_{0}^{1, p}\left(\mathbf{R}_{*}^{N}\right)$ is the completion of $C_{0}^{\infty}\left(\mathbf{R}_{*}^{N}\right)$ in the seminorm $\|u\|_{\mathcal{D}_{0}^{1, p}\left(\mathbf{R}_{*}^{N}\right)}=\|\nabla u\|_{p, \mathbf{R}_{*}^{N}}$. In this section, we give an estimate for the decay of solutions to equation (B.1) at the infinity. We have the following result.

Proposition B.1. Let $u$ be a solution of (B.1). Then there exists a constant $C>0$ such that

$$
|u(x)| \leq \frac{C}{1+|x|^{\frac{N-p}{p-1}}}, \quad \forall x \in \mathbf{R}_{*}^{N} .
$$

To prove Proposition B.1, the following preliminary estimate is needed.

Lemma B.2. Let $u$ be a solution of (B.1). Then there is a constant $C>0$ such that

$$
|u(x)| \leq \frac{C}{1+|x|^{\frac{N-p}{p}+\sigma}}, \quad \forall|x| \geq 1,
$$

for some $\sigma>0$.

The same estimate of Lemma B.2 was obtained in [6, Lemma B.3] for solutions to equation

$$
\left\{\begin{array}{l}
-\Delta_{p} u=|u|^{p^{*}-2} u \quad \text { in } \mathbf{R}^{N}, \\
u \in W^{1, p}\left(\mathbf{R}^{N}\right),
\end{array}\right.
$$

and in [31, Proposition 2.1] for solutions to more general equations. The proof of Lemma B.2 is the same as that of Lemma B.3 in [6] and of Proposition 2.1 in [31]. So we omit the details. To prove Proposition B.1, we will use the following comparison principle which is a special case of [31, Theorem 1.5]. Denote $\mathcal{D}^{1, p}(\Omega)=$ $\left\{u \in L^{p^{*}}(\Omega): \nabla u \in L^{p}(\Omega)\right\}$. 
Theorem B.3. Let $\Omega$ be an exterior domain such that $\Omega^{c}=\mathbf{R}^{N} \backslash \Omega$ is bounded and $f \in L^{\frac{N}{p}}(\Omega)$. Let $u \in \mathcal{D}^{1, p}(\Omega)$ be a subsolution of equation

$$
-\Delta_{p} u=f|u|^{p-2} u \text { in } \Omega \text {, }
$$

and $v \in \mathcal{D}^{1, p}(\Omega)$ a positive supersolution of

$$
-\Delta_{p} v=g|v|^{p-2} v \quad \text { in } \Omega,
$$

such that $\inf _{\partial \Omega} v>0$, where $g \in L^{\frac{N}{p}}(\Omega)$ satisfies $f \leq g$ in $\Omega$. Moreover, assume that

$$
\limsup _{R \rightarrow \infty} \frac{1}{R} \int_{B_{2 R}(0) \backslash B_{R}(0)}|u|^{p}|\nabla \log v|^{p-1} d x=0 .
$$

Then if $u \leq v$ on $\partial \Omega$, we have that

$$
u \leq v \quad \text { in } \Omega \text {. }
$$

Now we can prove Proposition B.1.

Proof of Proposition B.1. We will use Theorem B.3 to prove Proposition B.1. First we consider the case when $\mathbf{R}_{*}^{N}=\mathbf{R}^{N}$. Let $u$ be a solution to equation (B.1) with $\mathbf{R}_{*}^{N}=\mathbf{R}^{N}$ and set

$$
f(x)=\mu|u(x)|^{p^{*}-p}+\frac{|u(x)|^{p^{*}(s)-p}}{|x|^{s}} .
$$

Then $f \geq 0$. By Lemma B.2, we have

$$
f(x) \leq C|x|^{-\alpha} \text { for }|x| \geq 1,
$$

where

$$
\alpha=\min \left\{\left(p^{*}-p\right)\left(\frac{N-p}{p}+\sigma\right), s+\left(p^{*}(s)-p\right)\left(\frac{N-p}{p}+\sigma\right)\right\} .
$$

Since $0<s<p$ and $\sigma>0$, we have $\alpha=p+\left(p^{*}(s)-p\right) \sigma>p$. Thus $f \in$ $L^{\frac{N}{p}}\left(\mathbf{R}^{N} \backslash B_{1}(0)\right)$.

Let $\epsilon>0$ and write $\gamma=(N-p) /(p-1)$. Let $v(x)=|x|^{-\gamma}\left(1+|x|^{-\epsilon}\right)$ for $x \neq 0$. A simple calculation shows that

$$
-\Delta_{p} v=g(x) v^{p-1} \quad \text { for } x \neq 0,
$$

where

$$
g(x)=\frac{(p-1)(\gamma+\epsilon)^{p-1} \epsilon}{\left(1+|x|^{-\epsilon}\right)^{p-1}|x|^{p+(p-1) \epsilon}} .
$$

It is easy to derive from the above formula that

$$
C|x|^{-p-(p-1) \epsilon} \geq g(x) \geq C^{\prime}|x|^{-p-(p-1) \epsilon} \quad \text { for }|x| \geq 1,
$$

for $C, C^{\prime}>0$ depending on $N, p$ and $\epsilon$. As a result, we have $g \in L^{\frac{N}{p}}\left(\mathbf{R}^{N} \backslash B_{1}(0)\right)$ since $\epsilon>0$.

Choose $\epsilon>0$ small such that $p+(p-1) \epsilon<\alpha$. Then by (B.6) and (B.7), we can find a large number $R_{0}>1$ such that

$$
g(x) \geq f(x) \text { for }|x| \geq R_{0} .
$$


To verify (B.5), we note that $|\nabla \log v(x)| \leq C|x|^{-1}$ for $|x|$ large enough. Hence by Lemma B.2, we have for $R \geq R_{0}$ large enough,

$$
\begin{aligned}
\frac{1}{R} \int_{B_{2 R}(0) \backslash B_{R}(0)}|u|^{p}|\nabla \log v|^{p-1} d x & \leq \frac{C}{R} \int_{B_{2 R}(0) \backslash B_{R}(0)}|x|^{-\left(\frac{N-p}{p}+\sigma\right) p-(p-1)} d x \\
& \leq C R^{-p-\left(\frac{N-p}{p}+\sigma\right) p+N} \\
& =C R^{-p \sigma}
\end{aligned}
$$

which implies that (B.5) holds. Therefore, we can apply Theorem B.3 with $\Omega=$ $\mathbf{R}^{N} \backslash B_{R_{0}}(0)$ to conclude that

$$
u(x) \leq v\left(R_{0}\right)^{-1}\left(\sup _{|y|=R_{0}}|u(y)|\right) v(x) \quad \text { for }|x| \geq R_{0},
$$

where $v\left(R_{0}\right)=v(x)$ with $|x|=R_{0}$. We can prove the same estimate as above for $-u$ similarly. Therefore, we obtain that

$$
|u(x)| \leq C|x|^{-\frac{N-p}{p-1}} \quad \text { for }|x| \geq R_{0} .
$$

So we obtain an estimate for the decay of $u$ at infinity. To prove (B.2), it is enough to note that $u \in L_{\text {loc }}^{\infty}\left(\mathbf{R}^{N}\right)$, which can be proved by Moser's iteration method [25]. In particular, we obtain that

$$
|u(x)| \leq C^{\prime} \text { for }|x| \leq R_{0}
$$

for a constant $C^{\prime}>0$. Combining (B.8) and (B.9) yields (B.2). This finishes the proof of the case when $\mathbf{R}_{*}^{N}=\mathbf{R}^{N}$.

Next, consider the case when $\mathbf{R}_{*}^{N}=\mathbf{R}_{+}^{N}$. In this case, for any solution $u$, we use the odd extension

$$
\tilde{u}(x)= \begin{cases}u\left(x^{\prime}, x_{N}\right) & \text { if } x_{N} \geq 0, \\ -u\left(x^{\prime},-x_{N}\right) & \text { if } x_{N}<0,\end{cases}
$$

for $x=\left(x^{\prime}, x_{N}\right) \in \mathbf{R}^{N}$. It is direct to verify that $\tilde{u} \in \mathcal{D}^{1, p}\left(\mathbf{R}^{N}\right)$ and that $\tilde{u}$ is a solution of equation (B.1) with $\mathbf{R}_{*}^{N}=\mathbf{R}^{N}$. Thus our problem is reduced to the previous case. We then conclude easily that Proposition B.1 holds true for the second case. The proof of Proposition B.1 is complete.

\section{Appendix C. Estimates for solutions of $p$-Laplacian equations}

In this section, we copy two results on $p$-Laplacian equation from [6] without proof. We assume that $D$ is a bounded domain with $\Omega \subset \subset D$. The following result is Lemma 2.2 of [6].

Proposition C.1. For any functions $f_{1}(x) \geq 0$ and $f_{2}(x) \geq 0$, let $w \geq 0$ be the solution of

$$
\begin{cases}-\Delta_{p} w=f_{1}+f_{2} & \text { in } D, \\ w=0 & \text { on } \partial D .\end{cases}
$$

Let $w_{i}, i=1,2$, be the solution of

$$
\begin{cases}-\Delta_{p} w=f_{i} & \text { in } D \\ w=0 & \text { on } \partial D\end{cases}
$$


respectively. Then, there is a constant $C>0$, depending only on $r=\frac{1}{3} \operatorname{dist}(\Omega, \partial D)$, such that

$$
w(x) \leq C \inf _{y \in B_{r}(x)} w(y)+C w_{1}(x)+C w_{2}(x), \quad \forall x \in \Omega .
$$

Next result is Proposition C.1 of [6] which gives an estimate for solutions of $p$-Laplacian equation by Wolff potential.

Proposition C.2. There is a constant $\gamma \in(p-1,(p-1) N /(N-p+1))$, such that for any solution $u \in W^{1, p}(D) \cap L^{\infty}(D)$ to equation

$$
-\Delta_{p} u=f \quad \text { in } D,
$$

where $f \in L^{1}(D), f \geq 0$, there exists a constant $C=C(N, p, \gamma)>0$, such that for any $x \in D$ and $r \in(0$, dist $(x, \partial D))$,

$$
\left(f_{B_{r}(x)} u^{\gamma} d y\right)^{\frac{1}{\gamma}} \leq C+C \int_{r}^{\operatorname{dist}(x, \partial D)}\left(\frac{1}{t^{N-p}} \int_{B_{t}(x)} f d y\right)^{\frac{1}{p-1}} \frac{d t}{t} .
$$

\section{Appendix D. A global compactness result}

Recall that by (2.1) we define, for any function $u$,

$$
\rho_{x, \lambda}(u)=\lambda^{\frac{N-p}{p}} u(\lambda(\cdot-x))
$$

for any $\lambda>0$ and $x \in \mathbf{R}^{N}$. In this section, we give a global compactness result in the following proposition.

Proposition D.1. Let $u_{n}, n=1,2, \ldots$, be a solution of equation (1.7) with $\epsilon=\epsilon_{n} \rightarrow 0$, satisfying $\left\|u_{n}\right\| \leq C$ for some constant $C$ independent of $n$. Then $u_{n}$ can be decomposed as

$$
u_{n}=u_{0}+\sum_{j=1}^{k} \rho_{x_{n, j}, \lambda_{n, j}}\left(U_{j}\right)+\sum_{j=k+1}^{m} \rho_{0, \lambda_{n, j}}\left(U_{j}\right)+\omega_{n},
$$

where $u_{0}$ is a solution for (1.1), $\omega_{n} \rightarrow 0$ strongly in $W_{0}^{1, p}(\Omega), x_{n, j} \in \Omega$. And as $n \rightarrow \infty, \lambda_{n, j} \rightarrow \infty$ for all $1 \leq j \leq m, \lambda_{n, j} \operatorname{dist}\left(x_{n, j}, \partial \Omega\right) \rightarrow \infty$ for all $1 \leq j \leq k$. For $j=1,2, \cdots, k, U_{j}$ is a solution of

$$
\left\{\begin{array}{l}
-\Delta_{p} u=b_{j} \mu|u|^{p^{*}-2} u \quad \text { in } \mathbf{R}^{N}, \\
u \in \mathcal{D}^{1, p}\left(\mathbf{R}^{N}\right),
\end{array}\right.
$$

for some $b_{j} \in(0,1]$. For $j=k+1, k+2, \cdots, m, U_{j}$ is a solution of

$$
\left\{\begin{array}{l}
-\Delta_{p} u=b_{j} \mu|u|^{p^{*}-2} u+b_{j} \frac{|u|^{p^{*}(s)-2} u}{|x|^{s}} \text { in } \mathbf{R}_{*}^{N}, \\
u \in \mathcal{D}_{0}^{1, p}\left(\mathbf{R}_{*}^{N}\right)
\end{array}\right.
$$

for some $b_{j} \in(0,1]$, where $\mathbf{R}_{*}^{N}=\mathbf{R}^{N}$ when $0 \in \Omega$, and $\mathbf{R}_{*}^{N}=\mathbf{R}_{+}^{N}$ when $0 \in \partial \Omega$. Moreover, set $x_{n, i}=0$ for $i=k+1, \cdots, m$. For $i, j=1,2, \cdots, m$, if $i \neq j$, then

$$
\frac{\lambda_{n, j}}{\lambda_{n, i}}+\frac{\lambda_{n, i}}{\lambda_{n, j}}+\lambda_{n, j} \lambda_{n, i}\left|x_{n, i}-x_{n, j}\right|^{2} \rightarrow \infty
$$

as $n \rightarrow \infty$.

Proof. The proof is standard, see e.g. [6, 7, 32]. We omit the details. 


\section{References}

[1] Ambrosetti, A., and P. H. Rabinowitz: Dual variational methods in critical point theory and applications. - J. Funct. Anal. 14, 1973, 349-381.

[2] BAhri, A., and J. Coron: On a nonlinear elliptic equation involving the critical Sobolev exponent: the effect of the topology of the domain. - Comm. Pure Appl. Math. 41:3, 1998, 253-294.

[3] BrÉzIs, H.: Nonlinear elliptic equations involving the critical Sobolev exponent-survey and perspectives. - In: Directions in partial differential equations (Madison, WI, 1985), Publ. Math. Res. Center Univ. Wisconsin 54, Academic Press, Bonston, MA, 1987, 17-36.

[4] Brézis, H., and L. Nirenberg: Positive solutions of nonlinear elliptic equations involving critical Sobolev exponents. - Comm. Pure Appl. Math. 36:4, 1983, 437-477.

[5] Caffarelli, L. A., R. Kohn, and L. Nirenberg: First order interpolation inequalities with weights. - Compos. Math. 53:3, 1984, 259-275.

[6] CaO, D., S. Peng, and S. Yan: Infinitely many solutions for $p$-Laplacian equation involving critical Sobolev growth. - J. Funct. Anal. 262:6, 2012, 2861-2902.

[7] CAO, D., and S. YAN: Infinitely many solutions for an elliptic problem involving critical Sobolev growth and Hardy potential. - Calc. Var. Partial Differential Equations 38:3-4, 2010, 471-501.

[8] Capozzi, A., D. Fortunato, and G. Palmieri: An existence result for nonlinear elliptic problems involving critical exponents. - Ann. Inst. H. Poinceré Anal. Non Linéaire 2:6, 1985, 463-470.

[9] Cerami, G. S. Solimini, and M. Struwe: Some existence results for superlinear elliptic boundary value problems involving critical exponents. - J. Funct. Anal. 69:3, 1986, 289-306.

[10] Chern, J. L., and C.-S. Lin: Minimizers of Caffarelli-Kohn-Nirenberg inequalities with the singularity on the boundary. - Arch. Ration. Mech. Anal. 197:2, 2010, 401-432.

[11] Cingolani, S., and G. Vannella: Multiple positive solutions for a critical quasilinear equation via Morse theory. - Ann. Inst. H. Poincaré Anal. Non Liné aire 26:2, 2009, 397-413.

[12] Cingolani, S., and G. Vannella: On the multiplicity of positive solutions for $p$-Laplace equations via Morse theory. - J. Differential Equations 247:11, 2009, 3011-3027.

[13] Coron, J. M.: Topologie et cas limite des injections de Sobolev (Topology and limit case of Sobolev embeddings). - C. R. Acad. Sci. Paris Ser. I Math. 299:7, 1984, 209-212 (in French).

[14] Degiovanni, M., and S. Lancelotti: Linking solutions for $p$-Laplace equations with nonlinearity at critical growth. - J. Funct. Anal. 256:11, 2009, 3643-3659.

[15] Devillanova, G., and S. Solimini: Concentration estimates and multiple solutions to elliptic problems at critical growth. - Adv. Differential Equations 7:10, 2002, 1257-1280.

[16] Egnell, H.: Existence and nonexistence results for $m$-Laplace equations involving critical Sobolev exponents. - Arch. Ration. Mech. Anal. 104:1, 1988, 57-77.

[17] Ghoussoub, N.: Duality and perturbation methods in critical point theory. - Cambridge Tracts in Math. 107, Cambridge Univ. Press, Cambridge, 1993.

[18] Ghoussoub, N., and X. S. Kang: Hardy-Sobolev critical elliptic equations with boundary singularities. - Ann. Inst. H. Poincaré Anal. Non Linéaire 21:6, 2004, 767-793.

[19] Ghoussoub, N., and F. Robert: The effect of curvature on the best constant in the HardySobolev inequalities. - Geom. Funct. Anal. 16:6, 2006, 1201-1245.

[20] Ghoussoub, N., and C. Yuan: Multiple solutions for quasilinear PDEs involving critical Sobolev and Hardy exponents. - Trans. Amer. Math. Soc. 352:12, 2000, 5703-5743.

[21] Guedda, M., and L. Véron: Quasilinear elliptic equations involving critical Sobolev exponents. - Nonlinear Anal. 13:8, 1989, 879-902. 
[22] Han, Q., and F. H. Lin: Elliptic partial differential equations. - Courant Lect. Notes Math. 1, New York University, Courant Institute of Mathematical Sciences, New York, 1997.

[23] Hsia, C. H., C.-S. Lin, and H. Wadade: Revisiting an idea of Brézis and Nirenberg. - J. Funct. Anal. 259:7, 2010, 1816-1849.

[24] LI, Y. Y., and C.-S. Lin: A nonlinear elliptic PDE and two Sobolev-Hardy critical exponents. - Arch. Ration. Mech. Anal. 203:3, 2012, 943-968.

[25] Moser, J.: A new proof of De Giorgi's theorem concerning the regularity problem for elliptic differential equations. - Comm. Pure Appl. Math. 13, 1960, 457-468.

[26] Papageorgiou, E. H., and N.S. Papageorgiou: A multiplicity theorem for problems with the $p$-Laplacian. - J. Funct. Anal. 244:1, 2007, 63-77.

[27] Rabinowitz, P. H.: Variational methods for nonlinear eigenvalue problems. - In: Eigenvalues of non-linear problems (Centro Internaz. Mat. Estivo (C.I.M.E.), III Ciclo, Varenna) 1974, $139-195$.

[28] Rabinowitz, P. H.: Minimax methods in critical points theory with applications to differential equations. - CBMS Series 65, Providence, RI, 1986.

[29] Shen, Y., and X. Guo: On the existence of infinitely many critical points of the even functional $\int_{\Omega} F(x, u, D u) d x$ in $W_{0}^{1, p}$. - Acta Math. Sci. (English Ed.) 7:2, 1987, 187-195.

[30] Struwe, M.: A global compactness result for elliptic boundary value problems involving nonliearities. - Math. Z. 187:4, 1984, 511-517.

[31] XIANG, C.-L.: Asymptotic behaviors of solutions to quasilinear elliptic equations with critical Sobolev growth and Hardy potential. - J. Differential Equations 259:8, 2015, 3929-3954.

[32] YAN, S.: A global compactness result for quasilinear elliptic equations with critical Sobolev exponents. - Chinese J. Contemp. Math. 16:3, 1995, 227-234.

[33] YAN, S., and J. YANG: Infinitely many solutions for an elliptic problem involving critical Sobolev and Hardy-Sobolev exponents. - Calc. Var. Partial Differential Equations 48:3-4, 2013, 587-610.

[34] ZHU, X.: Nontrivial solution of quasilinear elliptic equations involving critical Sobolev exponent. - Sci. Sin. Ser. A 31:10, 1988, 1166-1181.

[35] Ziemer, W. P.: Weakly differentiable functions. - Grad. Texts in Math. 120, Springer-Verlag, New York, 1989.

Received 22 October $2015 \bullet$ Revised received 4 April $2016 \bullet$ Accepted 9 April 2016 